Revue d'histoire de l'Amérique française

Q6 REVUE D.HISTOIRE DE L'AMÉRIQUE FRANÇAISE

\title{
La sexualité comme pratique et rapport social chez les couples paysans du Saguenay (1860-1930)1
}

\section{Gérard Bouchard}

Volume 54, numéro 2, automne 2000

URI : https://id.erudit.org/iderudit/005337ar

DOI : https://doi.org/10.7202/005337ar

Aller au sommaire du numéro

\section{Éditeur(s)}

Institut d'histoire de l'Amérique française

\section{ISSN}

0035-2357 (imprimé)

1492-1383 (numérique)

Découvrir la revue

\section{Citer cet article}

Bouchard, G. (2000). La sexualité comme pratique et rapport social chez les couples paysans du Saguenay (1860-1930)1. Revue d'histoire de l'Amérique française, 54(2), 183-217. https://doi.org/10.7202/005337ar
Résumé de l'article

S'appuyant principalement sur plusieurs corpus de données orales, l'article analyse la sexualité dans le couple paysan saguenayen entre 1860 et 1930 en tant qu'elle s'inscrivait dans un important rapport social. Les données utilisées livrent des aperçus détaillés sur divers aspects de la sexualité avant et dans le mariage, notamment la grande ignorance chez les jeunes, la force des interdits et la flexibilité, sinon les contradictions, des règles morales selon qu'elles s'appliquaient à l'homme ou à la femme. Il en ressort que celle-ci était, de diverses façons, l'objet d'une violence psychologique et physique.Au chapitre de la contraception, l'analyse présente un éventail de techniques et moyens utilisés même en régime de fécondité dite naturelle. Elle démontre l'existence, chez la femme, d'une volonté précoce de limiter le nombre des naissances et d'opposer une certaine résistance à la norme sociale et morale. Mais cette volonté ne transparaît pas (ou très peu) dans les mesures du niveau de fécondité en raison du caractère généralement inefficace des recours utilisés.Le texte explore aussi le rapport social régissant les comportements sexuels dans le mariage. Une importante distinction est proposée entre la sexualité proprement dite, qui serait sous le contrôle de l'homme principalement (fréquence, modalités des rapports), et la procréation, dont la responsabilité relèverait surtout de la femme. Celle-ci se trouverait ainsi coincée entre les attentes du mari à l'échelle microsociale et les impératifs de l'Église et de l'État à l'échelle macrosociale. L'auteur pense qu'en définitive, la domination de la femme découlait plus de facteurs sociétaux que de la dynamique conjugale proprement dite.
Tous droits réservés @ Institut d'histoire de l'Amérique française, 2000

Ce document est protégé par la loi sur le droit d'auteur. L'utilisation des services d'Érudit (y compris la reproduction) est assujettie à sa politique d'utilisation que vous pouvez consulter en ligne.

https://apropos.erudit.org/fr/usagers/politique-dutilisation/ 


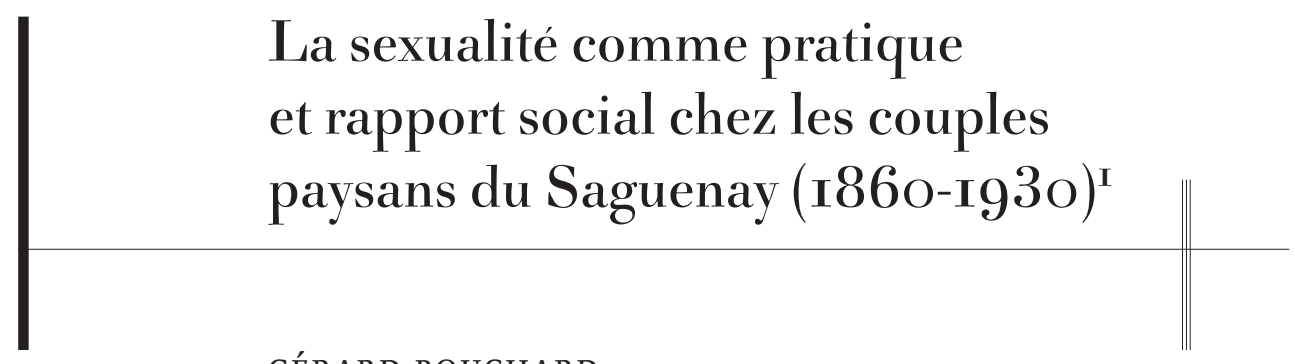

GÉRARD BOUCHARD

Institut interuniversitaire de recherches sur les populations (IREP)

Département des sciences humaines

Université du Québec à Chicoutimi

RÉSUMÉ • S'appuyant principalement sur plusieurs corpus de données orales, l'article analyse la sexualité dans le couple paysan saguenayen entre 1860 et 1930 en tant qu'elle s'inscrivait dans un important rapport social. Les données utilisées livrent des aperçus détaillés sur divers aspects de la sexualité avant et dans le mariage, notamment la grande ignorance chez les jeunes, la force des interdits et la flexibilité, sinon les contradictions, des règles morales selon qu'elles s'appliquaient à l'homme ou à la femme. II en ressort que celle-ci était, de diverses façons, l'objet d'une violence psychologique et physique.

Au chapitre de la contraception, l'analyse présente un éventail de techniques et moyens utilisés même en régime de fécondité dite naturelle. Elle démontre l'existence, chez la femme, d'une volonté précoce de limiter le nombre des naissances et d'opposer une certaine résistance à la norme sociale et morale. Mais cette volonté ne transparaît pas (ou très peu) dans les mesures du niveau de fécondité en raison du caractère généralement inefficace des recours utilisés.

Le texte explore aussi le rapport social régissant les comportements sexuels dans le mariage. Une importante distinction est proposée entre la sexualité proprement dite, qui serait sous le contrôle de l'homme principalement (fréquence, modalités des rapports), et la procréation, dont la responsabilité relèverait surtout de la femme. Celle-ci se trouverait ainsi coincée entre les attentes du mari à l'échelle microsociale et les impératifs de

1. Nos recherches ont pu être réalisées grâce à l'appui financier du Fonds FCAR (Québec), du Conseil de recherches en sciences humaines du Canada et de l'Université du Québec à Chicoutimi. L'auteur remercie tout particulièrement Denyse Baillargeon (en désaccord avec divers points de notre analyse) et Josée Gauthier qui ont bien voulu lire et commenter une version antérieure du texte. Josée Gauthier et Marc St-Hilaire ont aimablement transmis d'utiles informations tirées de leurs corpus de données orales. 
l'Église et de l'État à l'échelle macrosociale. L'auteur pense qu'en définitive, la domination de la femme découlait plus de facteurs sociétaux que de la dynamique conjugale proprement dite.

ABSTRACT - Relying mainly on many oral data corpuses, this paper analyses sexuality within married couples in peasant Saguenay during the period 1860-1930, insofar as sexual life was part of a major social relationship. The data yield detailed insights into various aspects of sexuality prior to and within the wedlock. The major traits brought out by the study include a deep ignorance of sexual matters among the newly-weds, the strength of taboos, along with the flexibility, not to say the contradictary nature, of moral rules according to whether they applied to men or to women. It follows that women were the target of psychological as well as physical violence.

Concerning contraception, the paper gives an array of techniques and devices that were in use even during this so-called natural fertility regime. It also documents the existence, among women, of a widespread willingness to reduce the number of births and to resist social and moral norms. However, given the inefficiency of most of the contraceptive techniques and devices available in the region at that time, this attitude was not echoed by the fertility level as reconstituted with standard measurements from the BALSAC population data bank.

The paper also addresses the social relationship governing the various sexual behaviors within the marriage. An important distinction is set forth between sexuality as such, which mostly fell under man's control (frequency of intercourse, etc.), and procreation, of which woman was primarily responsible. As a result, the wife was squeezed between the expectations of the husband (at the microsocial level) and the constraints of the Church and the State (at the macrosocial level). The author believes that, in the final analysis, the subjugation of women was the product more of societal factors than of conjugal dynamics.

\section{À partir d'une analyse de la sexualité, cet article veut éclairer un aspect 1 du rapport homme-femme dans la famille paysanne saguenayenne entre 1860 et $1930^{2}$. Nous nous proposons de présenter la vie sexuelle du couple paysan à la fois comme résultante et comme reflet d'un rapport so- cial. Ce rapport se reproduisait sur une double échelle : macrosociale d'abord, celle des structures sociétales, des classes, des pouvoirs institu- tionnels (Église, école, associations diverses), de l’État et du droit ; micro- sociale ensuite, à savoir l'univers familier de la vie conjugale et familiale, du voisinage et de la parenté, de la communauté immédiate.}

2. Voir aussi à ce sujet Gérard Bouchard, "Through the Meshes of Patriarchy: the Male/Female Relationship in the Saguenay Peasant Society (1860-1930)», The History of the Family: An International Quarterly, 4,4 (2000): 397-425. 


\section{LA SEXUALITÉ COMME FAIT SOCIAL}

Les comportements relevant de la sexualité revêtaient un caractère social au premier chef, dans la mesure où ils étaient l'objet de représentations partagées, de réglementations strictes, de surveillance étroite et de sanctions dans la collectivité. En outre, du moins dans le cas qui nous occupe, ils instituaient une relation entre deux personnes socialement définies dans le cadre conjugal, familial et communautaire. On peut donc étudier l'organisation de la vie sexuelle comme une sorte de microcosme structuré par un double rapport social : le couple conjugal et la vie sociétale (ce que les sociologues appellent parfois la société globale). Notre analyse de la sexualité au sein de la paysannerie saguenayenne veut contribuer à éclairer ce double rapport en conjuguant toutes les données empiriques que nous avons pu rassembler sur ce sujet.

Depuis plus de vingt ans, la sexualité, sous l'un ou l'autre de ses aspects, a pleinement intégré le champ de l'histoire sociale. On pourrait donner plusieurs références, allant des grands travaux de M. Foucault ${ }^{3}$ sur l'essor même de la sexualité comme objet scientifique et comme préoccupation morale, à l'étude de L. Gordon ${ }^{4}$ sur la diffusion des pratiques contraceptives aux États-Unis. En ce qui concerne le Québec, on est encore loin des grands ouvrages de synthèse comme celui que J. D’Emilio et E. B. Freedman ${ }^{5}$ ont consacré à l'histoire de la sexualité dans la société américaine. On possède toutefois de précieux aperçus pour la société bascanadienne grâce à l'ouvrage de S. Gagnon ${ }^{6}$, qui a su tirer un bon parti de quelques sources ecclésiastiques. Pour la fin du $\mathrm{XIx}^{\mathrm{e}}$ siècle et les premières décennies du $\mathrm{xx}^{\mathrm{e}}, \mathrm{D}$. Lemieux et L. Mercier ${ }^{7}$ ont eu accès à des confidences épistolaires, en milieu bourgeois principalement. Pour la première moitié du $\mathrm{xx}^{\mathrm{e}}$ siècle, A. Lévesque ${ }^{8}$ a exploré l'univers de la marginalité, avec ses

3. Michel Foucault, Histoire de la sexualité, 1 : La volonté de savoir (Paris, Gallimard, 1976); 2 : L'usage des plaisirs (Paris, Gallimard, 1984); 3 : Le souci de soi (Paris, Gallimard, 1984).

4. Linda Gordon, Woman's Body, Woman's Right. A Social History of Birth Control in America (New York, Grossman, 1976).

5. John D’Emilio, Estelle B. Freedman, Intimate Matters: A History of Sexuality in America (New York, Harper and Row, 1988).

6. Serge Gagnon, Plaisir d'amour et crainte de Dieu. Sexualité et confession au Bas-Canada (SainteFoy, Les Presses de l’Université Laval, 1990).

7. Denise Lemieux et Lucie Mercier, Les femmes au tournant du siècle, 1880-1940 : âges de la vie, maternité et quotidien (Québec, Institut québécois de recherche sur la culture, 1989).

8. Andrée Lévesque, La norme et les déviantes. Des fermmes au Québec pendant l'entre-deux-guerres (Montréal, Les Éditions du remue-ménage, 1989). 
violences et ses misères, pendant que $\mathrm{D}$. Baillargeon ${ }^{9}$ pénétrait la vie quotidienne de familles ouvrières montréalaises ${ }^{10}$. À ces apports pionniers auxquels se greffent quelques articles ${ }^{11}$, le présent essai veut ajouter un aperçu régional, centré sur la paysannerie du Saguenay ${ }^{12}$. Les travaux qui viennent d'être évoqués contiennent tous d'utiles renseignements sur les comportements sexuels, que les auteur(e)s intègrent à une problématique sociale de la condition féminine ou des rapports homme-femme. Adoptant une démarche semblable, nous nous sommes toutefois efforcé d'explorer plus avant l'univers des pratiques sexuelles en tirant profit, au fil des années, de plusieurs corpus de données orales (infra). Ainsi, la fréquentation de nombreuses personnes âgées, au cours des trente dernières années, nous a permis de percer, sur quelques points, le secret qui entourait habituellement les choses du sexe. Notre analyse a pu en outre s'appuyer sur des études détaillées de l'évolution de la fécondité saguenayenne et de la société paysanne entre 1842 et $1971^{13}$.

Le portrait global de la sexualité conjugale qui se dégage des travaux réalisés jusqu’ici sur le Québec pour la période qui nous concerne (18601930) montre un rapport très inégal qui met la femme en situation d'infériorité et de dépendance à l'endroit de son conjoint, de la communauté immédiate et des pouvoirs sociétaux. Ce portrait affiche toutefois des variations selon l'habitat (rural, urbain) et la classe sociale. On observe, par exemple, au sein de la bourgeoisie des signes précoces d'émancipation et de transgression des normes de l'Église catholique. Ce dernier phénomène mis à part, nous verrons que, pour l'essentiel, on retrouve une situation analogue dans l'ancienne paysannerie saguenayenne, mais avec des variantes assez significatives que nos données ont pu faire ressortir. Elles

9. Denyse Baillargeon, Ménagères au temps de la Crise (Montréal, Les Éditions du remueménage, 1991).

10. Sur les décennies ultérieures, voir Gaston Desjardins, L'amour en patience : la sexualité adolescente au Québec, 1940-1960 (Sainte-Foy, Les Presses de l'Université du Québec, 1995).

11. Par exemple Marie-Aimée Cliche, «Un secret bien gardé: l’inceste dans la société traditionnelle québécoise, 1858-1938", Revue d'histoire de l'Amérique française, 50,2 (automne 1996) : 201-226.

12. Nous y avons été précédé par Normande Simard-Vasil, Femmes, prise de décision et pouvoir. Analyse des relations entre les époux dans la famille chicoutimienne pour la première moitié du XXe siècle, mémoire de maîtrise (études régionales), Université du Québec à Chicoutimi, 1983, qui a réalisé (en partie sous notre direction) un mémoire de maîtrise sur le sujet en exploitant un corpus de 30 entrevues issues d'une entreprise collective (projet Filles de Maria).

13. Gérard Bouchard, Quelques Arpents d'Amérique. Population, économie, famille au Saguenay, 18381971 (Montréal, Boréal, 1996); Gérard Bouchard et Raymond Roy, «Fécondité et alphabétisation au Saguenay et au Québec ( $\mathrm{xIx}^{\mathrm{e}}-\mathrm{xx}^{\mathrm{e}}$ siècles)», Annales de démographie historique (1991) : 173-201. 
concernent, notamment, a) une volonté claire de résistance (mais qui ne trouvait guère à s'exprimer socialement) de la part de la femme dans ses rapports avec le prêtre, eu égard à la norme en matière de procréation; b) certains subterfuges pour déjouer les privilèges octroyés à l'époux; c) quelques désaccords dans la communauté locale, au sein de laquelle la femme trouvait des alliés; d) l'emprise de la religion et du prêtre sur la femme. En ce qui concerne la prise de décision au sein du couple, nous serons amené à distinguer soigneusement entre la procréation (qui relevait davantage de la femme) et la sexualité (qui relevait davantage de l'homme). Une autre distinction importante concerne la réduction de la fécondité, en tant que phénomène démographique mesurable, et le recours à des moyens contraceptifs qui pouvait demeurer sans effet observable sur les niveaux de fécondité. On verra aussi que, malgré les interdits, les tabous et les répressions dont elle était l'objet, la sexualité s'était néanmoins établie comme catégorie socioculturelle et comme sphère de comportement dotées d'une certaine autonomie. Enfin, nous aurons à examiner de près la question controversée de l'influence de l'Église ou du clergé sur les comportements procréatifs des couples, en particulier pour tout ce qui touche à la contraception.

Notre étude prendra une allure avant tout empirique, en essayant de tirer le maximum des sources utilisées. Celles-ci consistent en premier lieu dans le fichier de population BALSAC, utilisé pour l'analyse de la fécondité $^{14}$, ainsi qu'en de nombreuses monographies d'histoire locale. Mais nous avons surtout exploité plusieurs corpus de données orales, recueillies auprès de personnes âgées (hommes et femmes, en proportion à peu près égale, ayant au moins 75 ou 80 ans au moment des entretiens). Ce sont d'abord 150 entrevues en profondeur (d'une durée moyenne de deux heures environ) réalisées sous notre direction entre 1979 et 1995 et se rapportant en très grande partie au thème de cet essai, et un autre corpus de 140 entrevues à caractère plus général que nous avons effectuées personnellement entre 1968 et 1990 (la sexualité était l'un des douze thèmes abordés). Ce sont ensuite plus de 900 histoires de vie conservées aux Archives nationales du Québec à Chicoutimi (ANQC) et recueillies entre 1930 et 1980 (plusieurs d'entre elles témoignent des premières années du peuplement saguenayen, vers le milieu du $\mathrm{xIX}^{\mathrm{e}}$ siècle) par des membres

14. Sur cette banque de données, voir Gérard Bouchard, Raymond Roy, Bernard Casgrain, Michel Hubert, «Fichier de population et structures de gestion de base de données : le fichierréseau BALSAC et le système INGRES/INGRID», Histoire \& Mesure, 4,1/2 (1989): 39-57. 
de la Société historique du Saguenay. Ces entrevues, dactylographiées, sont d'une longueur très inégale (de cinq à trente pages, pour la plupart) et elles recouvrent un vaste éventail de sujets, dans un grand désordre. Elles contiennent plusieurs notations en rapport avec le thème de cet article (fréquentations, morale sexuelle, procréation, influence des prêtres, vie conjugale, etc.). Nous avons également utilisé une partie d'un corpus de 30 entrevues réalisées en 1982, avec notre assistance (conception du questionnaire, structure et suivi du projet), dans le cadre du projet Filles de Maria. Conduites auprès de femmes mariées entre 1921 et 1951, une dizaine de ces entrevues concernent le milieu paysan. Elles sont toutes centrées sur les comportements sexuels, la procréation et le rapport homme-femme. À cela s'ajoutent un corpus de 56 entretiens à caractère plus général, effectués en 1983 par Marc St-Hilaire auprès de personnes âgées, ainsi que des entrevues prolongées que nous avons pu réaliser au cours des dernières décennies à l'occasion de nombreuses rencontres avec une douzaine de personnes âgées, qu'on peut considérer ici comme des témoins privilégiés. Nous avons pu en effet établir un rapport très intime avec ces individus que nous avons fréquentés pendant plusieurs années, ce qui nous a permis d'explorer plus à fond l'univers de la sexualité conjugale. Enfin, nous avons mis à profit quelques recueils saguenayens d'histoires de vie ${ }^{15}$ ainsi que les premiers résultats d'une thèse de doctorat en cours (Josée Gauthier) sous notre direction et portant sur le rituel de la naissance ${ }^{16}$. Quelques autres sources seront signalées en cours de route notamment un corpus de 300 entrevues sur le rituel du mariage, réalisées à l'échelle du Québec dans le cadre d'un projet de l'IREP ${ }^{17}$.

Tous ces entretiens ont été conduits tantôt avec des hommes ou avec des femmes, tantôt avec des couples. Certains corpus ont été confectionnés à l'aide de questionnaires rigides, d'autres résultent d'entretiens moins encadrés. Dans un cas comme dans l'autre, et comme nous aurons l'occasion de le rappeler plus loin, la quantification n'était guère possible, et ce pour diverses raisons, dont, principalement, le caractère hétérogène des corpus,

15. Notamment ceux qui ont été publiés en 1978, 1980 et 1990 par l'AFEAS du Saguenay (Dans l'histoire... des femmes aussi... au Saguenay-Lac-Saint-Jean, I (Chicoutimi, Éditions Sciences Moderne, 1978); Dans l'histoire... des femmes aussi... au Saguenay-Lac-Saint-Jean, II (Chicoutimi, Éditions Sciences Moderne, 1980); Dans l'histoire des femmes aussi au Saguenay-Lac-Saint-Jean, III (Chicoutimi, Éditions JCL, 1990).

16. Voir aussi Josée Gauthier, Évolution des pratiques coutumières entourant la naissance au Saguenay et dans Charlevoix (1900-1950), mémoire de maîtrise (études régionales), Université du Québec à Chicoutimi, 1991.

17. Projet réalisé sous la direction de Gérard Bouchard, Anne-Marie Desdouits et René Hardy. 
le petit nombre des entrevues dans plusieurs corpus et, dans les gros corpus, le caractère trop épars des notations. On comprendra que la matière ne se prêtait guère à des entretiens très systématiques, sur le modèle des enquêtes sociologiques; il faut garder à l'esprit l'époque à laquelle plusieurs corpus ont été réalisés, les interdits qui pesaient sur les sujets abordés, les réticences éprouvées par la plupart des personnes interviewées. Nous avons essayé de réaliser une synthèse de toutes ces données, en nous éclairant de ce que trente ans de recherche nous ont appris sur l'histoire de la paysannerie saguenayenne. Nous pensons que la diversité et l'ampleur des sources exploitées donnent une assurance raisonnable que les déficiences habituelles, inhérentes aux données orales, ont pu être surmontées en grande partie (oublis, omissions volontaires, distorsions, erreurs, rationalisations, amplifications de traits remarquables mais exceptionnels, informatrices intimidées par un conjoint...). Dans le traitement que nous en avons fait, nous avons cherché à contourner ces carences en mettant l'accent sur les données les plus factuelles, validées par divers recoupements.

En somme, la multiplicité des sources présente l'inconvénient de l'hétérogénéité, mais elle comporte aussi le double avantage d'enrichir la base empirique et d'offrir au chercheur la possibilité de valider les informations; le poids des convergences s'avère ici très précieux. Par ailleurs, l'hétérogénéité en elle-même n'est pas si gênante, dans la mesure où notre analyse est largement descriptive. Dans cet esprit, n'y avait-il pas intérêt à tirer profit de toutes les sources disponibles, dans un domaine de recherche où elles sont si rares?

Ainsi constituées, la majeure partie de nos données portent sur la période 1900-1930. Cependant, plusieurs personnes interrogées ont témoigné également sur la génération qui les a précédées (en gros : celle de leurs père et mère, oncles et tantes). D'autres entrevues avec des personnes nées à une époque relativement récente (1900-1905 par exemple) ont pu être utilisées pour éclairer la période préconjugale et les premières années du mariage, et aussi pour les informations qu'elles contiennent sur les âges antérieurs (enfance, adolescence).

\section{UNE SOCIÉTÉ ET SES NORMES}

L’environnement normatif en matière de sexualité était d'abord défini par le magistère de l’Église catholique. De très bonnes études ont été consacrées à ce sujet, au Québec et ailleurs ${ }^{18}$, et nous nous contenterons d'y

18. Pour une perspective générale, voir par exemple John Thomas Noonan, Contraception: A History of its Treatment by the Catholic Theologians and Canonists (Cambridge, Mass., Harvard 
référer étant donné que, sous ce rapport, le diocèse de Chicoutimi ne présente guère d'éléments de spécificité. La religion catholique avait le souci de sacraliser la sexualité en l'élevant à une très haute valeur morale qui convenait à sa finalité première : la procréation. Elle acceptait aussi (ou plus exactement tolérait) le plaisir qui lui était associé, mais à condition que les couples s'efforcent de s'en détacher afin de conférer au lien qui les unissait une qualité de plus en plus spirituelle. Le corollaire le plus immédiat de cet enseignement était une incitation à la famille nombreuse, puisqu'aucun moyen artificiel ne devait faire obstacle à la procréation.

Mais il s'ensuivait aussi un certain nombre d'aménagements, propositions ou règles dont la logique est moins apparente. D’abord, la femme était particulièrement identifiée aux choses du sexe, dans lesquelles on lui attribuait une responsabilité prééminente - par exemple, en glorifiant la maternité et les devoirs qui y étaient attachés. Deuxièmement, en matière de sexualité, c'est la femme qui devait se mettre au service de l'homme, dont elle devait satisfaire les besoins sans jamais se dérober (sauf exception). En troisième lieu, l'univers de la sexualité était ordinairement synonyme d'impureté et, en ce sens, il inspirait de la répulsion. L’image de la femme vierge/souillée, mère/impure, reflète les deux figures contradictoires de cet univers. En quatrième lieu, la vocation associée à la procréation conduisait à statuer qu'en situation d'incompatibilité (dans les accouchements principalement), la vie de l'enfant devait prévaloir sur celle de la mère ${ }^{19}$. Dans le cadre de cet essai, nous prendrons simplement acte de ces données, sans nous interroger sur leur origine lointaine ou sur leur

University Press, 1965); Anita Caron, dir., Femmes et pouvoir dans l'Église (Montréal, VLB éditeur, 1991). En ce qui a trait au Québec : Michèle Jean, «Féminisme et religion au Québec, 1900-1978», dans Elisabeth-J. Lacelle, dir., La femme et la religion au Canada français (Montréal, Éditions Bellarmin, 1979), 33-42; Isabelle Rodrigue, Limiter les naissances: entre le modèle véhiculé par l'École sociale populaire et la réalité des couples québécois (1920-1940) (Sainte-Foy, Les Presses de l'Université Laval, coll. «Les Cahiers de recherche du GREMF», nº 73, 1996), mémoire de maîtrise (histoire), Université Laval, 1993. Consulter également Andrée Lévesque, op. cit., chap. II et III.

19. On trouvera diverses formulations de ces conceptions dans bien des textes devenus des classiques du genre, notamment Alexis Mailloux, Manuel des parents chrétiens ou Devoirs des pères et mères dans l'éducation religieuse de leurs enfants (Québec, A. Côté, 1851); Joseph D’anjou, «La famille a le droit et le devoir d'être féconde», Mission et droit de la famille (1959) : 79-80; les recueils produits par les Semaines sociales du Canada français (1924 : «La famille»; 1940 : «Le chrétien dans la famille et la nation"), etc. Voir aussi Colette Carisse, Planification des naissances en milieu canadien-français (Montréal, Presses de l'Université de Montréal, 1964); La famille : mythe et réalité québécoise. Rapport présenté au Conseil des Affaires sociales et de la famille (Québec, Éditeur officiel du Québec, 1974); Denyse Baillargeon, op. cit., chap. 6; Serge Gagnon, «Pour une histoire du sexuel et du sacral en régime catholique ", Annales de Bretagne et des Pays de l'Ouest, 95,4 (1988): 435-446; id., Plaisir d'amour et crainte..., op. cit. 
portée symbolique. M. Albert-Llorca ${ }^{20}$, par exemple, a commenté la difficile prise en charge de la féminité par la chrétienté, l'idéal de la pureté virginale contredisant celui de la procréation. D’autres auteurs ont tenté de montrer les racines profondes de l'ascétisme chrétien ou puritain. Pour $\mathrm{M}$. Mason ${ }^{21}$, notamment, la morale victorienne tire son origine de l'idéologie des Lumières qui célébrait le règne de la raison et l'empire qu'elle devait exercer sur le corps, comme condition de la liberté. Récupérée, déviée par le protestantisme, l'idée aurait ensuite inspiré la morale victorienne. D'autres ${ }^{22}$, dans la tradition de M. Foucault, ont suggéré que la pratique de la sexualité se serait pervertie au cours du xvIII ${ }^{\mathrm{e}}$ siècle, en devenant objet d'angoisse, de peurs, de restrictions morales.

D'une façon ou d'une autre, toutes ces représentations étaient au cœur de la culture paysanne saguenayenne. La femme devait s'astreindre à la plus grande sobriété dans le vêtement (robes longues), la tenue (absence totale de maquillage, cheveux longs), le langage. Comme ailleurs, un tabou frappait la sexualité, qui était bannie des conversations ordinaires et même de l'éducation des jeunes gens. Dans la vie communautaire (à l'église, à l'école, dans les associations), les garçons et les filles étaient séparés; ils arrivaient au mariage très ignorants, au terme de fréquentations étroitement surveillées. La prédication de l'Église inhibait la sexualité : les deux époux devaient «se respecter comme des temples sacrés ${ }^{23}$ ». Les mères enceintes cachaient leur grossesse le plus longtemps possible. La femme, bien plus que l'homme, était particulièrement marquée. Ne disait-on pas : «une personne du sexe»? Dispensatrice des plaisirs et dépositaire des mystères du sexe, elle était objet de convoitises mais aussi de suspicions. Si l'homme venait à commettre l'adultère, c'était bien sa faute à elle, qui n'avait pas su «lui donner son besoin».

Le cadre juridique, en particulier le droit criminel, faisait écho aux interdits de l'Église en frappant d'illégalité l'avortement, la diffusion d'informations relatives à la contraception, la vente d'instruments ou d'articles contraceptifs (cette loi du $\mathrm{xIx}^{\mathrm{e}}$ siècle ne fut abolie qu'en 1968). Les élites

20. Marlène Albert-Llorca, «Les fils de la Vierge. Broderie et dentelle dans l'éducation des jeunes filles», L'Homme, 133 (1995): 99-122.

21. Michael Mason, The Making of Victorian Sexual Attitudes (New York, Oxford University Press, 1994).

22. Roy Porter, Lesley Hall, The Facts of Life: The Creation of Sexual Knowledge in Britain, 16501950 (New Haven, Yale University Press, 1995).

23. Échos paroissial (publié par les pères eudistes de la paroisse du Bassin de Chicoutimi), septembre 1911, 132. 
politiques et socioculturelles s'associaient aussi à la promotion de la morale et des idéaux de l'Église catholique. La plupart des notables voyaient dans la fécondité élevée un rouage important de la survivance nationale, adhérant à ce que plusieurs, depuis 1918, ont appelé la «revanche des berceaux ${ }^{24}$ ». D'après A. McLaren et A. T. McLaren ${ }^{25}$, ce n'est pas avant les années 1930 que se firent vraiment entendre au Québec (en l'occurrence à Montréal) les premiers laïques promoteurs de la contraception ${ }^{26}$. Enfin, dans un cadre plus immédiat qui est celui de la communauté, les femmes devaient composer avec les normes et les attentes de la parenté, du voisinage, de la paroisse, généralement en consonance avec les règles de l’Église et du droit.

Nous examinons maintenant la réalité au jour le jour des comportements reliés à la sexualité, pour accéder ensuite à une réflexion sur les relations ou interactions entre les normes et les actes.

\section{LA SEXUALITÉ AU QUOTIDIEN}

Un mot d'abord sur le niveau de fécondité de ces couples paysans. Durant la période considérée, l'ensemble des couples ont enregistré en moyenne de 10 à 11 naissances vivantes. En termes statistiques, cette moyenne est d'autant plus significative qu'elle est proche du mode; c'est dire qu'elle reflète le comportement de la grande majorité des couples. On relève en effet que trois quarts des mariages étaient suivis de sept naissances vivantes et plus (cette mesure tient compte des couples stériles). Peu de filles échappaient à ce modèle; jusqu'au milieu du $\mathrm{xx}^{\mathrm{e}}$ siècle, le taux de

24. Voir la critique que nous avons faite de cette thèse dans Gérard Bouchard et Richard Lalou, «La surfécondité des couples québécois depuis le $\mathrm{xVII}^{\mathrm{e}}$ siècle : essai de mesure et d'interprétation", Recherches sociographiques, 34,1 (1993) : 9-44; Gérard Bouchard, Quelques arpents..., op. cit., chap. VIII. On trouvera dans le Collectif Clio, L'histoire des femmes au Québec depuis quatre siècles (Montréal, Quinze, 1982), Andrée Lévesque, op. cit. et quelques autres ouvrages des références aux principaux porte-parole de l'idéologie des notables que furent Henri Bourassa, Hermas Bastien, Esdras Minville, C.-J. Magnan et autres.

25. Angus McLaren et Arlene Tigar McLaren, The Bedroom and the State: The Changing Practices and Politics of Contraception and Abortion in Canada, 1880-1980 (Toronto, McClelland and Stewart Limited, 1986).

26. On sait qu’à la même époque au Canada anglais, une partie des élites se laissait convaincre du bien-fondé des pratiques contraceptives, mais par souci d'eugénisme social, pour empêcher de se reproduire des éléments de la société jugés indésirables, et non pas en vertu d'une reconnaissance des droits des femmes. Voir Dianne Dodd, «The Canadian Birth Control Movement on Trial, 1936-1937», Histoire sociale/Social History, 16,32 (novembre 1983) : 411-428. 
célibat féminin (à 50 ans et plus) a oscillé entre $4 \%$ et $6 \%{ }^{27}$. Toutefois, c'est une tout autre question de savoir dans quelle mesure ces chiffres reflètent l'emprise de la religion ou du clergé sur les couples. Nous y reviendrons. Ajoutons que les gens mariés après 1930 ont été les premiers à pratiquer la contraception à une échelle significative. Mais comme il s'agissait d'une contraception d'arrêt ${ }^{28}$, ses effets ne se sont pas manifestés avant les années $1940^{29}$.

Essayons d'éclairer la vie sexuelle sous-jacente à cette statistique de la procréation. On note d'abord que, surtout pour les filles, l'apprentissage de la sexualité dans la famille ou à l'école était pratiquement inexistant jusqu'au mariage. La très grande majorité d'entre elles (plus des quatre cinquièmes assurément) ignoraient presque tout (une informatrice déclare : "On savait même pas qu'on savait rien", corpus J. Gauthier). Sauf exception, les mères évitaient ce sujet dans leurs conversations avec leurs filles, souvent même après leur mariage. C'est entre femmes d'un certain âge seulement qu'on en parlait, et dans la plus grande discrétion. Les filles qui possédaient quelques informations les avaient ordinairement obtenues de leurs sœurs aînées ou d’amies plus âgées. Mais même dans ce cas, les renseignements diffusés étaient plus qu'élémentaires : il fallait «faire l'acte» pour devenir enceinte, il fallait devenir enceinte ("partir en famille») pour avoir un bébé, etc. Et parmi ces "privilégiées», très peu semblent avoir su exactement ce qu'était l'«acte» lui-même. Les autres ignoraient à peu près tout de l'amour physique, de la grossesse et des modalités de la naissance. On ne permettait pas aux enfants ni aux jeunes gens d'entrer dans l'étable lorsqu'on faisait saillir les animaux ou lorsque les vaches mettaient bas ${ }^{30}$. À plus forte raison, on s'assurait que les enfants, sous quelque prétexte, fussent toujours éloignés de la maison au moment d'un accouchement. Ils apprenaient ensuite que le corbeau ou les Sauvages avaient apporté un bébé. Si un enfant avait perçu les signes de la grossesse chez sa mère, il aurait été mal avisé de l'interroger à ce sujet. Cela dit, il semble que les garçons aient été un peu moins ignorants

27. Soulignons que le modèle saguenayen ne paraît pas exceptionnel, à la lumière des données présentement disponibles sur d'autres paysanneries du Québec à la même époque (Gaspésie, Bas-Saint-Laurent, Charlevoix, Estrie, Mauricie, Verchères...).

28. Pendant une bonne partie de leur vie fertile, ces couples procréaient donc au même rythme que les autres, mais ils cessaient un peu avant que la femme ait atteint la ménopause.

29. Sur tout ce qui précède, voir Gérard Bouchard, Quelques arpents..., op. cit., chap. VII.

30. Une informatrice, après son mariage seulement, se fit expliquer par son mari ce qui se passait à l'étable — où elle n'était toujours pas admise à ces occasions (corpus G. Bouchard). 
que les filles, parfois. Certains apprenaient des bribes d'informations aux chantiers forestiers, à la faveur des conversations du camp. D’autres, en séjour de travail à Québec, à Montréal ou aux États-Unis, pouvaient tirer profit d'occasions ou de circonstances qui ne se présentaient pas dans leur paroisse. Mais dans l'ensemble, une grande ignorance régnait chez les uns comme chez les autres.

La plupart des filles ne découvraient la réalité brutale des menstruations qu'au moment où celles-ci commençaient à se manifester. Un grand nombre d'entre elles, effrayées et perturbées par cette expérience, la vivaient pendant plusieurs mois ou même quelques années dans le silence et dans de terribles angoisses, convaincues qu'elles étaient atteintes d'une maladie honteuse, mystérieuse, dont elles allaient mourir peut-être. Celles qui, n'en pouvant plus, s'avisaient de s'en ouvrir à leur mère se heurtaient souvent à un mutisme qui ajoutait aux tourments. Parfois une sœur aînée, mariée, venait au secours de la jeune fille, ou bien le prêtre au confessionnal ${ }^{31}$. Que les filles n'aient pas choisi d'échanger ces informations et de s'entraider davantage démontre la force du tabou qui pesait sur ce sujet. Laissée à elle-même, chacune veillait à "s'arranger" avec des bouts de tissus ou de vieux linges qui servaient de serviettes et que, secrètement, elle allait laver au ruisseau ou à la rivière; ou bien elle les mettait à tremper dans un seau d'eau caché derrière la porte de sa chambre, etc.

Le mariage était la porte d'entrée dans l'univers trouble, souvent aussi redouté que désiré, de la vie sexuelle. Et le seuil n’en était pas franchi aisément. La nuit de noces, en particulier, était un cauchemar pour la majorité. Fréquemment, l'union n'était pas consommée dès la première nuit, par pudeur, par crainte ou par incapacité physique. Très rares étaient les jeunes mariés qui, en cette occasion, offraient leur nudité à la vue de leur conjoint; les jeunes femmes, en particulier, se réfugiaient plutôt dans la salle de bains, derrière la porte ou même dans la garde-robe (penderie) pour enfiler leur robe de nuit. Certaines, au moment de «se faire étrenner» par leur mari, découvraient avec un peu de dégoût que les hommes étaient poilus, ou s'effrayaient de la dimension de leur sexe. D’autres croyaient qu'en mariage on faisait l'amour une fois l'an, ou que la femme avait un enfant chaque fois qu'elle faisait l'«acte». Il n'était pas rare qu'au lendemain d'une nuit peu glorieuse, une jeune mariée se rendit au

31. Au cours des cinq dernières années, nous avons interrogé sur le même sujet une quinzaine de femmes âgées de 50 à 60 et ayant vécu dans le monde paysan; nous avons été surpris d'y retrouver, sous une forme atténuée, plusieurs traits de la situation que nous décrivons. 
presbytère en quête de clarifications. Mais ni l'homme ni la femme ne s'ouvraient aisément de ces questions à leurs proches.

Devenues enceintes, la plupart des jeunes conjointes s'interrogeaient sur leur état et sur la suite des choses, découvrant au fur et à mesure l'arrêt des menstruations, les nausées, les maux digestifs, les somnolences, les seins qui gonflent, les premiers signes de vie du fotus, les eaux qui "crèvent "... Étrangement, encore là, on relève peu de consultations auprès des aînées, sauf aux derniers stades de la grossesse. Pour certaines, c'était même péché que d'aborder ce sujet. Par scrupule, les mères se bandaient ou se corsetaient («se corsaient») le ventre et portaient force jupes, jupons et tabliers plissés pour dissimuler leur état. Il semble qu’à leur première expérience, la majorité d'entre elles ignoraient la durée d'une grossesse; certaines croyaient qu'il fallait continuer à avoir des relations sexuelles pour nourrir le fotus (corpus J. Gauthier); d'autres, que l'enfant naîtrait par le nombril, et le reste ${ }^{32}$.

Les rapports de nature sexuelle avant le mariage, sous quelque forme que ce soit, étaient très peu fréquents et, sauf exception, tenaient à peu de chose. Dans les veillées de jeunesse, pour éviter la promiscuité, les parents s'assuraient que les chaises soient suffisamment espacées les unes des autres pour laisser passer une personne, ainsi que le voulait la règle. Deux conjoints se souviennent qu'en sept ans de fréquentations, ils s'étaient embrassés sept fois, et toujours en présence de parents. Une jeune fille qui s'était fait embrasser sur la joue a cru devenir enceinte. Les fréquentations se déroulaient sous haute surveillance; les instants d'intimité étaient rares ${ }^{33}$. Au village, il était interdit aux jeunes gens de patiner le soir; certains curés menaçaient de refuser l'absolution aux contrevenants ${ }^{34}$. Quelques prêtres se montraient moins sévères mais, dans tous les cas, les patineurs n'avaient droit à aucun contact physique; il leur fallait se tenir par les deux bouts d'un bâton dont la longueur était réglementée (de douze à dix-huit pouces). Après la messe dominicale, il était interdit aux garçons et filles de se rassembler en des lieux publics comme

32. Dans ce contexte d'ignorance, un savoir s'instituait néanmoins, fait de croyances ou de superstitions les plus diverses. Elles sont analysées dans la thèse de doctorat (en cours) de Josée Gauthier.

33. Comme l'attestent les recherches effectuées sur le Saguenay en rapport avec le rituel du mariage, dans le cadre du projet de l'IREP mentionné plus haut (voir par exemple Marie-Josée Huot, Les pratiques rituelles entourant le mariage dans les régions du Saguenay et de Charlevoix, mémoire de maîtrise (études régionales), Université du Québec à Chicoutimi, 1991.

34. Par exemple : Pierre-L. Côté, Saint-Félicien : son histoire religieuse (Saint-Félicien, La Fabrique de la paroisse de Saint-Félicien, 1984), 121. 
le bureau de poste. Il était particulièrement important que la future conjointe se présentât vierge à son conjoint. Et pour prouver sa vertu au prétendant, elle était avertie de repousser toutes ses avances jusqu'au jour du mariage. Encore là, la fille et le garçon n'étaient pas tout à fait soumis à la même norme. L'homme se voyait plus facilement pardonner ses faiblesses : il était dit naturellement impulsif, entraîné par sa virilité, ses instincts, sujet aux débordements de la chair. La femme devait voir à les contenir dans les limites de la morale.

On relève tout de même un certain nombre de dérogations aux règles, les plus visibles étant les conceptions prénuptiales et les naissances hors mariage. Mais dans les deux cas, les comparaisons que nous avons pu effectuer mènent à la conclusion que les proportions saguenayennes étaient basses $^{35}$. Les premières n'affectaient que de $3 \%$ à $4 \%$ des naissances entre le milieu du $\mathrm{XIX}^{\mathrm{e}}$ siècle et la fin de notre période. Quant aux deuxièmes, leur proportion (déclarée) oscillait autour de $1 \%$. Dans les deux cas, on relève certains facteurs de sous-estimation, mais peu importants. Quoi qu'il en soit, ces résultats sont concordants avec les indices très élevés de la conformité religieuse, telle qu'elle a pu être mesurée à partir des rapports annuels des curés, à l'aide d'indicateurs comme la pratique pascale, l'assistance hebdomadaire à la messe, l'observance du repos dominical et d'autres. Pendant toute la période, les valeurs prises par tous ces indices sont supérieures à $90 \%{ }^{36}$.

Le portrait qui se dégage de ces observations demeure difficilement compréhensible si l'on ne tient pas assez compte du complexe de peur, d'angoisse, d'interdit, de culpabilité et de séduction/répulsion qui composait le discours et la symbolique de la sexualité. C’est dans cette perspective qu'il faut également considérer les pratiques sexuelles au sein du couple. Nous nous pencherons d'abord sur les pratiques les plus légitimes; les autres seront abordées dans la partie suivante. L'acte sexuel se déroulait dans la plus grande simplicité, sans préalable, ni fantaisie ni variation («il n'y avait qu'une façon de faire ça», corpus G. Bouchard). Les "agréments» n'étant pas bien vus (ni très connus), on allait droit à l'essentiel, toute l'affaire étant expédiée en quelques minutes, et parfois moins. Les partenaires gardaient leurs vêtements de nuit et apprenaient à ouvrer en silence,

35. Gérard Bouchard, «L'évolution des conceptions prénuptiales comme indicateur de changement culturel», Annales de démographie historique (1993) : 25-49.

36. Pascale Dupont, Conformité et déviance : la pratique religieuse au Saguenay, 1886-1951, mémoire de maîtrise (études régionales), Université du Québec à Chicoutimi, 1995. 
étant donné la promiscuité domestique. Sauf exception, l'acte n'était pratiqué que le soir, au lit, alors que toute la maisonnée s'était retirée pour la nuit. À cause des circonstances qui entouraient l'acte sexuel (caractère expéditif, perspective d'une autre grossesse...), plusieurs femmes (la moitié peut-être, selon nos sources, mais il est difficile de juger) n'éprouvaient pas d'orgasme, et certaines aucun plaisir. La majorité faisaient l'amour par devoir d'abord, plusieurs dans la crainte et la contrainte.

La fréquence des relations sexuelles était parfois quotidienne (jusqu’à deux fois par jour), parfois hebdomadaire, la moyenne se situant vraisemblablement à deux fois par semaine environ. Selon quelques témoignages, elle augmentait durant les premiers mois de la grossesse : à partir du moment où elle savait qu'elle était enceinte, la femme n'avait plus à se soucier de retarder la prochaine naissance ("on se lâchait», corpus G. Bouchard). Pour la même raison, la fréquence des rapports s'accélérait durant les premières années qui suivaient la ménopause. Toutefois, la période des relevailles, qui suivait l'accouchement, était marquée par l'abstinence ${ }^{37}$ : chose exceptionnelle, la femme était alors autorisée à se refuser à son conjoint. Cette période durait en principe 40 jours mais, en pratique, elle était très souvent abrégée, surtout à cause de l'impatience du mari. Une règle semblable s'appliquait durant les menstruations, mais celle-ci ne souffrait guère de dérogations (on croyait, par exemple, qu'un enfant conçu dans ces circonstances allait naitre avec un strabisme).

La vie sexuelle conjugale consacrait la domination de l'homme, sanctionnée par la norme de l'Église et le consensus social. C'est l'homme qui prenait l'initiative et menait l'opération. La passivité de la femme était inscrite dans la position des partenaires : l'homme au-dessus, sa conjointe au-dessous. Pourtant, la majorité des femmes n'acceptaient pas de bon cœur la subordination dans laquelle elles étaient tenues. Elles prenaient peu de plaisir à l'acte sexuel, d'abord à cause de son caractère expéditif, comme nous l'avons signalé, mais aussi et surtout parce qu'il était associé à la possibilité d'une autre grossesse. En général, cette perspective était considérée négativement et elle contribuait fort à compromettre les rapports de la femme avec la sexualité conjugale. Néanmoins, les conjointes s'accommodaient tant bien que mal de cet ordre de choses, et ce pour quatre raisons. Les deux premières ont trait aux croyances religieuses et aux pressions exercées par les prêtres ainsi que par la famille et la

37. Il faudrait entendre ici continence. Mais nous avons choisi de nous en remettre à l'expression utilisée par nos informateurs et informatrices. 
communauté (ce sujet sera abordé plus loin). Une troisième raison tenait à ce que, si les besoins sexuels de l'homme n'étaient pas satisfaits, il serait peut-être tenté d' "aller voir ailleurs"; alors, le désordre s'installerait dans le couple, la conjointe en serait tenue responsable et la honte s'abattrait sur elle. Une dernière raison, invoquée par quelques informatrices, relevait de la compassion que la femme éprouvait pour son mari, qui «méritait bien d'avoir ses petits plaisirs» (corpus G. Bouchard). Une bonne partie des femmes (il est impossible de quantifier plus précisément) faisaient donc l'amour par devoir, en silence. Il n'y avait guère là matière à discussion ou à négociation. Le couple n'abordait à peu près pas ce sujet ("on ne pensait pas rien qu’à ça»), pas plus qu’on échangeait ou dialoguait sur le reste de la vie émotive : les sentiments, les perceptions, les attentes, «on n’avait pas le temps de parler de ça».

D’une certaine façon, toutes ces données s'accordaient avec le genre de vie de la paysannerie saguenayenne, qui était fait de privations et de duretés auxquelles étaient assujettis aussi bien les enfants que les hommes et les femmes. La règle du primum vivere dans un contexte de peuplement dressait les personnes à la plus grande sobriété et leur faisait accepter des conditions de vie très difficiles ("on n'avait jamais connu autre chose»). Dans le cas des femmes, la grossesse n’était pas tenue pour une maladie et elles devaient continuer à exécuter leurs tâches quotidiennes jusqu'à l'accouchement ${ }^{38}$. On faisait peu pour soulager les maux de la femme enceinte; cela aurait même été immoral, selon quelques informatrices : il fallait laisser faire la nature. Des femmes acceptaient (ou étaient contraintes) d'accoucher avec un minimum d'assistance dans des installations de fortune, aux chantiers d'hiver par exemple.

\section{PRATIQUES CONTRACEPTIVES, MÈRES SUBVERSIVES ?}

Établissons tout de suite une donnée générale : durant la période considérée, la contraception, comme modèle de comportement collectif et comme recours délibéré, rationnel, ayant pour effet de réduire significativement la fécondité, n’existait pas dans cette paysannerie. Ainsi que nous l'avons indiqué, les indices réputés les plus fiables et les plus sensibles pour détecter ce genre de pratiques ${ }^{39}$ montrent qu'elles ont commencé à se manifester durant la décennie 1940 seulement, sous la forme d’une contra-

38. Josée Gauthier, op. cit., chap. 3.

39. Par exemple, l'indice de Ansley J. Coale et T. James Trussell, «Technical Note : Finding the True Parameters That Specify a Model of Marital Fertility", Population Index, 44 (1978) : 201-213. 
ception d'arrêt, chez les couples mariés après $1930^{40}$. Avant cette période, les indices démographiques ne décèlent aucune forme de contraception, qu'il s'agisse d'intervalle protogénésique (délai écoulé entre le mariage et la première naissance), d'espacement (délai moyen entre les naissances subséquentes) ou d'arrêt (supra). Du point de vue de l'histoire sociale, démographique ou économique, ce résultat est extrêmement significatif. Toutefois, au regard de l'histoire socioculturelle, il doit être interprété avec prudence. En effet, ce que l'analyse statistique établit avec certitude, c'est que la fécondité naturelle a persisté jusqu’à la décennie 1940; mais il serait erroné d'en inférer qu'avant cette date, les couples saguenayens ne faisaient pas de tentatives (infructueuses) pour réduire le nombre des enfants ou qu'il n'existait pas de volonté contraceptive dans cette population. C'est justement ce que démontrent nos données qualitatives.

Les corpus que nous avons analysés révèlent clairement que les femmes et les couples paysans désiraient restreindre le nombre des naissances et utilisaient divers recours à cette fin. Mais ces derniers se situaient dans les limites de ce qui était socialement et culturellement possible et ils s'avéraient inefficaces. Parmi les recours (instruments ou procédés) relevés au Saguenay, on distingue des moyens dits naturels (par exemple l'allaitement, l'abstinence), d'autres de nature mécanique ou chimique ou encore d'autres relevant de la médecine à proprement parler. Mais ce qu'il importe surtout de souligner, c'est qu'aucun ne peut être assimilé à ce qu'on appelle couramment les moyens indirects de contraception, lesquels consistent, par exemple, à hausser l'âge au mariage, à favoriser les mariages entre conjoints d'âges très inégaux (surtout entre femmes âgées et jeunes hommes) ou à encourager le célibat définitif. Les recours qui ont été mentionnés le plus souvent dans nos corpus de données sont les suivants :

- Prolonger l'allaitement afin d'étirer la période d'infertilité qui suit habituellement l'accouchement. Cette pratique très ancienne avait cours en Europe au moins depuis le XVIII ${ }^{\mathrm{e}}$ siècle et sans doute depuis le Moyen Âge ${ }^{41}$. Elle était courante au Saguenay également, où la relation entre l'allaitement prolongé et la stérilité provisoire était bien connue des femmes. D’après les témoignages disponibles, les

40. Gérard Bouchard et Raymond Roy, loc. cit.; Gérard Bouchard, Quelques arpents..., op. cit., chap. VIII.

41. Sølvi Sogner, "Allaitement au sein et abstinence sexuelle au Moyen Âge», Annales de démographie historique (1986) : 353-375; Marie-France Morel, «Théories et pratiques de l’allaitement en France au xviII ${ }^{e}$ siècle", Annales de démographie historique (1976) : 393-427; Étienne Van De Walle et Francine Van De Walle, «Allaitement, stérilité et contraception : les opinions jusqu'au XIX ${ }^{\mathrm{e}}$ siècle », Population, 4-5 (juillet/octobre 1972) : 685-701. 
femmes allaitaient une douzaine de mois en moyenne (ce qui correspond à la durée estimée chez les femmes canadiennes aux XVII ${ }^{\mathrm{e}}$ et $\mathrm{XVIII}^{\mathrm{e}}$ siècles ${ }^{42}$ ). Les valeurs extrêmes relevées vont de trois à trente mois (et même davantage, si l'on en croit quelques informatrices). Certes, la notion de "prolongement» est très relative. Il semble cependant bien établi que la majorité des femmes auraient sevré l'enfant plus tôt, n'eût été la motivation que suscitaient les vertus contraceptives prêtées à l'allaitement. Cet énoncé est validé par les nombreux témoignages de femmes affirmant qu'elles avaient allaité le plus longtemps possible, mais avaient dû finalement sevrer leur bébé pour diverses raisons : les travaux domestiques ne leur en laissaient plus le temps, les dents de l'enfant les blessaient, les menstruations avaient repris, l'enfant ne voulait plus téter, elles étaient redevenues enceintes, l'enfant était trop grand, c'était devenu "gênant", etc. ${ }^{43}$. Il est vrai que sur le plan médical, l'allaitement prolongé était encouragé pour donner une meilleure alimentation à l'enfant ou pour purifier le sang de la mère. Mais l'intention contraceptive était omniprésente. Les femmes en parlaient discrètement à la veillée. Les mères encourageaient leurs filles mariées à s'y adonner (surtout à partir de leur quatrième ou cinquième accouchement). Des informatrices nées à la fin du XIx ${ }^{\mathrm{e}}$ siècle ou au début du $\mathrm{xx}^{\mathrm{e}}$ assurent que cette pratique était très répandue du temps de leur mère et même de leur grand-mère. Dans un de nos corpus de données, sur 32 femmes (ayant répondu à la question), la moitié allaitaient encore lorsqu'elles redevenaient enceintes ${ }^{44}$. Souvent, leurs menstruations ne reprenaient pas; elles cessaient d'avoir du lait et, bientôt, constataient la nouvelle grossesse. Quoi qu'il en soit, il faut éviter de surestimer les effets contraceptifs de l'allaitement : ces femmes n'en avaient pas moins une dizaine d'enfants en moyenne.

- S’abstenir de tout rapport sexuel. Pour des raisons médicales, économiques ou autres, des conjoints vivaient pendant quelque temps «comme frère et sœur». Dans certains cas, cette situation a duré quelques années.

- Interrompre le coït avant l'éjaculation. On disait «faire l'amour à côté», «s'arrêter en chemin", "décharger son voyage à côté», "se graisser la bedaine», "commencer dans le salon, terminer dans la cuisine ${ }^{45}$ ". Ce recours est men-

42. Richard Lalou, La haute fécondité des Québécoises avant la révolution contraceptive : effet de nature ou effet de culture? Un bilan des connaissances, Document II-C-179 de l'IREP, 1990.

43. Certains enfants étaient assez vieux pour pouvoir dégrafer eux-mêmes le corsage de leur mère.

44. Notons qu'au Saguenay, la morale catholique et paysanne n'interdisait pas les rapports sexuels durant la période d'allaitement, contrairement à ce qu'on observait dans certains pays d'Europe (Étienne et Francine Van De Walle, loc. cit.).

45. Une variante de cette dernière expression, tirée du corpus Filles de Maria, a aussi été rapportée par Danielle Gauvreau et Peter Gossage, «Empêcher la famille : fécondité et contraception 
tionné dans nos données, mais il était apparemment beaucoup moins répandu que les deux précédents.

- Aussitôt après l'acte, marcher vivement ou sautiller près du lit "pour faire descendre la semence» ou "faire tomber le germe». Ce recours a été mentionné par six ou sept informatrices. Certaines ont toutefois affirmé qu'il était très répandu, mais les femmes étaient "gênées d’en parler» par crainte d'être tournées en ridicule (l'une d'elles a eu 14 enfants, les autres 10 ou 12...). Ce procédé n'est pas une invention saguenayenne; deux informateurs nés avant 1900 nous l'ont rapporté pour la région de Charlevoix. Il a été signalé également chez les anciens Égyptiens, les aborigènes d'Australie, certaines populations africaines et autres.

- Procéder à la toilette du vagin après l'acte. Ce moyen, qui est une variante du précédent, a été mentionné à quelques reprises.

- Uriner après l'acte. C'est une deuxième variante. Quelques femmes en avaient fait une habitude.

- Provoquer l'évacuation de l'embryon (ou du fotus?) au premier stade de la grossesse. Ce résultat pouvait être atteint de diverses façons : a) se livrer à des travaux très durs aux champs ou à la maison (comme laver les planchers); dans ce dernier cas, on disait : "elle est partie en grand ménage" (corpus G. Bouchard); b) marcher beaucoup; c) descendre lourdement («débouler») les escaliers; d) casser du bois de chauffage en sautant dessus (corpus J. Gauthier); e) passer de longues heures à cueillir des fruits sauvages, en position accroupie; f) monter à cheval; g) se masser vivement le ventre; h) prendre des bains à la moutarde ou au vinaigre; i) manger un peu de savon; j) porter des vêtements très serrés à la taille. Mentionnons aussi que, parmi les recettes de médecine populaire relevées dans des enquêtes ethnographiques, plusieurs avaient pour fonction de déclencher les menstruations ou de provoquer une fausse couche ${ }^{46}$. Mais nos corpus ne font pas état d'infanticides ni d'avortements pratiqués à l'aide d'aiguilles ou autrement à un stade avancé de la grossesse (on ne doit pas en conclure que de tels incidents ne sont pas survenus).

au Québec, 1920-60", Canadian Historical Review, 78,3 (septembre 1997) : 478-510, qui ont réexploité le corpus d'entretiens constitué par Denyse Baillargeon, op. cit. («On fumait dans le salon et on crachait dans la cuisine»).

46. Francine Saillant, Ginette Côté, Serge Genest, Se soigner en famille. Les recettes de médecine populaire dans les familles québécoises du début $d u x^{*}$ siècle (Québec, Centre de recherche sur les services communautaires, Université Laval, 1990), I ; Francine Saillant et Hélène Laforce, «Médecine domestique et pratiques sociales entourant la reproduction chez les Québécoises», Canadian Folklore Canadien, 15,2 (1993) : 31-50. 
- L'ablation de l'utérus et/ou des ovaires. Plusieurs informatrices ont rapporté ce recours à titre contraceptif, le présentant comme étant bien connu des femmes. En particulier chez celles qui éprouvaient des difficultés pendant la grossesse, l'hystérectomie (la "grande opération») et l'ovariectomie (la "petite opération») étaient vivement souhaitées, comme une "délivrance». Mais il fallait que le médecin les prescrive. Dans le corpus des Filles de Maria, une femme sur trois dit avoir subi l'une ou l'autre opération ${ }^{47}$.

Les autres moyens de contraception alors en usage dans d'autres populations ${ }^{48}$ étaient peu connus et ne semblent pas avoir été utilisés dans les campagnes saguenayennes avant 1930. C’est le cas des méthodes thermique et cyclique (Ogino-Knauss), qui n’ont été mises au point que dans les années 1920. C'est également le cas du condom, qu'on ne trouvait pas dans les pharmacies locales et qui n’était jamais évoqué dans la presse régionale, sous forme d'encarts publicitaires par exemple ${ }^{49}$. Il en est de même pour les potions "délivrantes», gelées, pessaires et mousses spermicides.

Dans l'un des corpus que nous avons nous-même constitués, 22 des 34 femmes interrogées disaient ne pas avoir connu l'existence de moyens contraceptifs à l'époque où elles ont eu leurs enfants. Mais cette information est trompeuse, dans la mesure où la majorité d'entre elles avaient pratiqué l'allaitement prolongé et certaines avaient eu provisoirement recours à l'abstinence ${ }^{50}$. Ce qu'il importe surtout de retenir, c'est l'existence d'une volonté contraceptive assez répandue qui s'est manifestée de diverses façons, même si les moyens utilisés étaient plutôt inefficaces (comme l'atteste, encore une fois, le taux élevé et stable de la fécondité durant la période). Ils demeuraient sans résultat pour deux raisons. Sur le plan technique d'abord, plusieurs étaient tout simplement déficients. Sur le plan social et culturel ensuite, ils faisaient tous l'objet d'interdictions et

47. L'énumération qui précède n'est sans doute pas complète et son contenu n'est sûrement pas original. Sous une forme ou sous une autre, on retrouve la plupart de ces recours dans diverses sociétés. Pour le Canada anglophone, par exemple, voir Alison Prentice, Paula Bourne, Gail Cuthbert Brandt, Beth Light, Wendy Mithchinson, Naomi Black, Canadian Women : A History (Toronto, Harcourt Brace Jovanovich, 1988), 164-165. Pour la Russie, A. G. Vichnevski, "Le rôle des connaissances historiques dans l'étude du comportement procréateur en U.R.S.S. », Annales de démographie historique (1987) : 213-239.

48. Aux États-Unis par exemple : Janet Farrell Brodie, Contraception and Abortion in NineteenthCentury America (Ithaca, Cornell University Press, 1994).

49. Nous avons lu attentivement les hebdomadaires saguenayens entre 1880 et 1930 .

50. Ce paradoxe indique peut-être qu'au moment des entrevues, ces informatrices se référaient à une définition moderne de la contraception, celle qui comporte l'utilisation du condom, du stérilet ou de la pilule. Mais il peut s'agir aussi d'une simple rationalisation destinée à chasser le mauvais souvenir d'actes jadis tenus pour immoraux. 
d'une étroite surveillance. Ainsi, l'allaitement prolongé, tenu pour naturel par l'Église, était toléré mais dans certaines limites. Dès qu'il devenait clair que l'enfant pouvait être sevré sans risque pour sa santé (à 12 ou à 18 mois par exemple), la mère s'exposait à une remontrance de la part du curé. C'était la même chose pour l'abstinence, qui n'était permise que dans des circonstances particulières ("quand il y a une raison grave», écrivait en 1923 le sulpicien et futur recteur de l'Université de Montréal Olivier Maurault $\left.{ }^{51}\right)$. Le coït interrompu était lui aussi formellement interdit, de même que toute mesure ayant pour but d' "extraire la semence» après qu'elle a été introduite dans le «vase féminin». Étaient ici visées les ablutions vaginales ainsi que l'habitude de se lever pour marcher ou sautiller après l'acte. Ce procédé était, pour certaines informatrices (ex. : corpus G. Bouchard, J. Gauthier), objet de scandale. On tenait en effet que, dès après l'éjaculation, l'enfant était formé. En conséquence, les femmes qui s'adonnaient au sautillement «se trouvaient à pilasser leur enfant» à peine conçu. Quant à l'ablation des ovaires et/ou de l'utérus, le médecin ne pouvait la prescrire qu'avec la permission du curé et les cas de refus étaient très fréquents, même lorsque la vie de la mère était mise en péril. Enfin, on devine que toutes les tentatives visant à expulser artificiellement ou à détruire le «fruit» étaient condamnées avec la plus grande sévérité. C'était l'une des raisons - parmi d'autres — pour lesquelles les femmes redoutaient tant les fausses couches : elles craignaient que la communauté n'y voie un geste délibéré de leur part et c'est un autre motif pour lequel les femmes tenaient à garder leur grossesse secrète le plus longtemps possible. Ainsi, en cas d'avortement prématuré (ce qui était fréquent), elles s'épargnaient les calomnies et n'encouraient pas la honte du voisinage.

\section{POURQUOI LES RÉSISTANCES À LA CONTRACEPTION ?}

En ce qui concerne la fécondité saguenayenne et la contraception avant 1940, trois questions se posent, qu'il importe de bien distinguer : Pourquoi cette fécondité était-elle aussi élevée? Pourquoi s'est-elle maintenue à un haut niveau, alors que divers facteurs tendaient à la faire chuter? Qu'estce qui a finalement provoqué son déclin? Résumons d'abord brièvement les réponses que nous avons élaborées ailleurs ${ }^{52}$. La fécondité était élevée pour les raisons suivantes : la famille nombreuse était relativement peu coûteuse (les enfants étant peu scolarisés), elle était rentable (les enfants

51. Voir Isabelle Rodrigue, op. cit., 90.

52. Gérard Bouchard, Quelques arpents..., op. cit., chap. VIII et XIX. 
fournissaient la main-d'œuvre sur la ferme et rapportaient du numéraire à la famille en s'employant à des travaux hors ferme), les larges réseaux de parenté constituaient un indispensable filet social pour les parents vieillissants (particulièrement en contexte de peuplement), la procréation était une vieille tradition inscrite au cœur de la culture paysanne ${ }^{53}$. À compter du début $\mathrm{du} \mathrm{xx}^{\mathrm{e}}$ siècle, divers facteurs auraient pu compromettre la fécondité naturelle, par exemple : la saturation progressive des espaces agricoles, le déclin régulier du pluriétablissement ${ }^{54}$, les réticences des femmes à l'endroit des naissances répétées. Mais d'autres facteurs agissaient en sens contraire. Les enfants adultes ou mariés continuaient de se mettre au service de leurs parents, alors même que ceux-ci ne pouvaient plus, en retour, les établir sur des terres; en d'autres mots, le service familial se poursuivait même s'il était amputé de sa réciprocité économique ou matérielle. La fécondité élevée pouvait donc demeurer rentable pour les parents, en plein contexte de saturation foncière. En outre, l'emprise de la religion et les pressions exercées par les prêtres contribuaient à contrer les réticences des couples, en particulier des femmes (infra), et à fermer l'accès aux moyens contraceptifs. Enfin, la domination exercée par l'homme dans le rapport conjugal allait dans le même sens.

Pour ce qui est de la troisième question, nous avons proposé à ce sujet un modèle composite, multifactoriel, qui fait intervenir des facteurs locaux ou régionaux (l'achèvement de l'occupation du terroir comme agent déstabilisateur, la fin de la réallocation, le déclin de la pluriactivité, la relaxation de la morale traditionnelle après les années 1940, l'apparition des politiques sociales gouvernementales qui ont rendu les parents moins dépendants de leurs enfants), mais aussi des facteurs extrarégionaux comme la scolarisation obligatoire qui a élevé le coût de la famille nombreuse, la diffusion de nouveaux modèles culturels par l'entremise des médias (imprimés, radio...), la lente émancipation des femmes, l'apparition de nouveaux procédés contraceptifs (méthodes thermique, cyclique) dont l'Église s'est peu à peu accommodée, enfin toutes les transformations associées à la Crise de 1929 et à la Deuxième Guerre mondiale.

Revenons, à la lumière de nos données, sur deux facteurs qui ont empêché - et par conséquent retardé — le déclin de la fécondité naturelle en dépit des éléments qui la menaçaient.

53. À dessein, nous ne faisons pas mention de la supposée revanche des berceaux comme facteur explicatif. Ce modèle nous parait en effet peu fondé, pour des raisons qui ont été exposées ailleurs (voir supra, note 24).

54. Le fait, pour un couple paysan, de pouvoir établir plus d'un enfant comme cultivateur. 


\section{A. L'influence de la religion et des prêtres}

Le premier de ces facteurs concerne l'emprise de la religion et les pressions exercées par les prêtres. Idéalement, on voudrait pouvoir évaluer en elle-même l'emprise du sacré, en dehors de tout appareil de contrôle ou de répression; mais les données ne le permettent guère ${ }^{55}$. On ne peut que la déduire des effets que produisaient les interventions du clergé sur les couples, et surtout sur les femmes. Au Saguenay, si on en juge par les témoignages disponibles, ces effets étaient très importants. Dans leurs sermons dominicaux, la majorité des curés abordaient assez régulièrement le sujet de la natalité pour rappeler aux femmes leur devoir sacré. Rien ne devait entraver la procréation, pas même une maladie qui aurait mis la vie de la mère en danger : si elle en mourait, elle aurait une plus belle place au ciel, elle serait assise plus près du Bon Dieu ${ }^{56}$. Par contre, aller contre la nature, c'était l'enfer assuré, avec ses flammes éternelles et toutes ses horreurs. Lors des retraites fermées que les femmes - tout comme les hommes, mais séparément - devaient fréquenter une fois l'an pendant une semaine, des prédicateurs professionnels (prêtres dits réguliers, membres de communautés) se chargeaient d'évoquer le sort effroyable des délinquantes. Les informatrices se rappellent plus particulièrement les prédicateurs qui piquaient de saintes colères, frappaient du poing sur la chaire, hurlaient dans l'église. Des femmes sortaient en pleurs, prises de frayeur, et en perdaient le sommeil. Chez celles-là, une profonde culpabilité s'installait : «À tant se faire chauffer les oreilles, on était sûre qu'on était méchante» (corpus G. Bouchard). Les prédicateurs expliquaient qu’à leur mort, les femmes auraient à rendre compte à Dieu des enfants qu'elles n'avaient pas eus et des plaisirs qui les avaient remplacés. Des informatrices racontent que durant les semaines et les mois qui suivaient, "ça décollait enceintes dans la paroisse ${ }^{57}$ ». Des entretiens conduits auprès d'hommes âgés ont livré le même genre de témoignages, même si les hommes, encore une fois, se sentaient moins concernés (corpus G. Bouchard, J. Gauthier; entrevues ANQC).

55. À part quelques énoncés stéréotypés : «Les enfants, c’est au bon plaisir de Dieu », "Qui vient viendra», "C'est la Providence qui mène ça», etc.

56. Plusieurs curés parlaient régulièrement du sexe, soit pour y inciter en quelque sorte les parents chrétiens suivant l'idéal de la procréation, soit pour en éloigner les jeunes gens; mais toujours avec la même vigueur.

57. Sur ce qui précède, les témoignages sont très nombreux dans tous les corpus. Dans celui des Filles de Maria par exemple, presque toutes les informatrices ont dit avoir été influencées par la prédication des prêtres. 
L'action de la chaire se prolongeait dans l'intimité du confessionnal où les curés posaient les questions embarrassantes, recevaient les aveux timorés, rappelaient les règles sacrées de l'Église, faisaient pression sur celle-ci ou sur celle-là, et prononçaient les sanctions appropriées. Quelques-uns enjoignaient tout particulièrement aux pénitentes de voir à bien conserver la "sève" après l'acte (ce qui semble attester la popularité de certains moyens contraceptifs évoqués plus haut). Les futures conjointes venues se confesser avant la cérémonie nuptiale étaient informées que, désormais, leur corps ne leur appartenait plus et elles étaient instamment invitées à faire leurs devoirs envers leur mari. Trop ignorantes, un grand nombre ne comprenaient même pas le sens exact de ces paroles et n'osaient le demander. En d'autres circonstances toutefois, le prêtre se faisait plus explicite. Devant celles qui avaient sciemment empêché ou retardé la vie et qui en étaient parfois à leur seconde offense, il faisait entendre la colère de Dieu : admonestées, elles se voyaient refuser l'absolution et l'accès à la communion. Quelques informatrices ont ainsi raconté avoir été «discommuniées" (c'était l'expression courante) pour avoir fait chambre à part après un douzième ou un quinzième enfant, pour avoir fait l'amour "à côté » après que le médecin les eut mis en garde contre une autre grossesse, pour avoir retardé une naissance en allaitant jusqu'à deux ou trois ans ${ }^{58}$. Enfin, les rencontres à domicile effectuées dans le cadre de la visite paroissiale annuelle ou pour réconforter un malade fournissaient au curé une autre occasion de veiller à l'état des mœurs et des ventres. Ce sujet a été traité par de nombreux auteurs et nous avons peu à y ajouter. Ordinairement, si le dernier-né avait commencé à marcher et que la femme n'était pas encore redevenue enceinte, le curé y allait d'une petite remarque à l'intention de la mère : qu'est-ce qu'elle attendait pour se remettre à l'ouvrage? Il n'y avait donc plus de place dans cette grande maison? etc. Un curé arpentait les pièces, comptait les lits vides; un autre avertissait qu'il réservait un espace blanc dans son «livre des âmes» pour l'année suivante. Une jeune femme qui s'adonnait à la lecture se faisait dire : «Tu ferais mieux de faire des enfants» (corpus G. Bouchard). Ces

58. Tous ces témoignages de femmes à propos du confessionnal ont été confirmés par des entrevues que nous avons pu réaliser avec trois vieux prêtres de la région (qui ont demandé à ne pas être identifiés). L'univers qui nous a été ainsi dévoilé ressemble beaucoup à celui qui fut décrit par un confesseur (catholique) polonais au début du $\mathrm{xx}^{\mathrm{e}}$ siècle. Voir Eli Zaretsky, «Female Sexuality and the Catholic Confessional ", Signs : Journal of Women in Culture and Society, 6,1 (automne 1980): 176-184. 
interventions étaient souvent faites amicalement, et parfois durement, mais elles étaient toujours prises au sérieux.

Il est important de signaler une certaine diversité dans la manière et même dans le jugement (plus ou moins sévère) des prêtres. Il n'était pas rare qu'une femme, se jugeant trop sévèrement punie dans sa paroisse, trouve grâce (et absolution) auprès d'un curé voisin, plus indulgent, plus sensible au sort des mères. Quoi qu'il en soit, il est assuré que le discours et les pressions exercées par le prêtre dans la vie paroissiale étaient efficaces, ayant pour effet de refréner le recours aux moyens contraceptifs déjà disponibles à l'époque - si élémentaires fussent-ils. "Je n'étais pas pour me damner juste pour avoir un enfant de moins", commente une femme qui en a eu 14 (corpus G. Bouchard) ${ }^{59}$. Une analyse de régression multiple ${ }^{60}$ a effectivement fait ressortir le poids du facteur religieux, celuici étant mesuré à l'aide du délai moyen écoulé entre la naissance et le baptême des enfants (on peut considérer que ce délai, commandé par une croyance religieuse profonde et très ancienne ${ }^{61}$, reflétait l'intensité de la foi). Parmi les facteurs commandant les comportements à l'endroit de la fécondité, la religion se classait au deuxième rang derrière l'alphabétisation, mais devant les variables économiques ${ }^{62}$.

Sur le plan social, soulignons l'appui et même la complicité que les mères rencontraient auprès de plusieurs médecins en désaccord avec le curé. Ces situations étaient fréquentes, au Saguenay comme (apparemment) ailleurs au Québec : le médecin déconseillait formellement une autre grossesse qui, pour une raison ou pour une autre, mettrait la vie de la femme en danger, mais le prêtre enjoignait à cette dernière de passer outre, sous peine d'excommunication et de damnation. Selon la logique de l’Église, la femme devait redevenir enceinte aussi souvent et aussi longtemps que la Providence le commandait, peu importe l'issue de la grossesse. Dans les cas les plus graves, certains médecins informaient

59. La vigilance exercée par les pasteurs et l'écoute qu'ils trouvaient auprès des familles expliquent sans doute que les évêques du diocèse n'aient pas cru utile d'intervenir souvent sur ce sujet dans leurs lettres et mandements (durant la période considérée).

60. Gérard Bouchard, Quelques arpents..., op. cit., 455-460.

61. On pensait que si le nouveau-né décédait avant d'être baptisé, il séjournerait éternellement dans les limbes, d'où la hâte que l'on mettait à le présenter aux fonts baptismaux (les parents se représentaient les limbes comme un lieu de malheur où les enfants étaient privés éternellement de la vue du Bon Dieu).

62. L'analyse statistique de la relation fécondité/religion est complexe. Dans leur enquête sur la fécondité des Québécoises, Jacques Henripin et Évelyne Lapierre-Adamcyk, dans La fin de la revanche des berceaux. Qu'en pensent les Québécoises? (Montréal, Presses de l’Université de Montréal, 
discrètement l'intéressée des meilleurs moyens à prendre pour se tirer d'affaire, ou bien la dirigeait vers un prêtre plus conciliant. Après 1930, des médecins diffusaient des informations sur la méthode rythmique, ou encore prescrivaient carrément une hystérectomie (souvent de complaisance); le prêtre devait alors s'incliner, même s'il était souvent sceptique.

\section{B. La relation homme-femme dans le mariage}

Le deuxième point concerne la relation homme-femme dans le mariage. Il est certain que la pratique sexuelle était dominée par l'homme. Sauf durant la période des relevailles, les femmes n'avaient pas le droit de se refuser à leur mari. Certaines se livraient à d'innocents stratagèmes dont l'efficacité reste douteuse. Ainsi une informatrice, au moment de se mettre au lit, prétextait ne pas avoir complété son rosaire quotidien et s'agenouillait près du lit en espérant que son mari finisse par s'endormir (elle eut 18 enfants en 22 ans - corpus G. Bouchard). À cause de la perspective de la grossesse et des maux qui l'accompagnaient, les rapports sexuels perdaient beaucoup d'attrait pour la femme. Mais la morale conjugale étant impérative, il n'y avait guère à discuter et, de toute façon, l'ère n'était pas au dialogue entre les conjoints. Ils n'abordaient presque jamais ce sujet entre eux, pas plus qu'ils n'échangeaient sur leurs sentiments intimes («on ne se bâdrait pas de ça», disent plusieurs informatrices). Mais en réalité, au-delà de son mari, la femme était davantage captive d'un pouvoir social incarné par le clergé. C’est elle surtout qui servait de médiation entre la religion et la fécondité. C’est elle qui était particulièrement interpellée par le prêtre, lequel en faisait la cible privilégiée de ses interventions en chaire, au confessionnal ou ailleurs. C'est elle aussi qui était tenue princi-

1974), concluaient que l'appartenance religieuse (catholique, protestante ou autre) ne contribuait pas à expliquer la fécondité élevée parmi les femmes nées entre 1906 et 1920; mais ils arrivaient à une conclusion contraire concernant les femmes nées entre 1931 et 1935. Ces résultats sont difficiles à expliquer, étant donné ce que l'on sait de l'évolution culturelle du Québec francophone à cette époque (la première cohorte de femmes a été davantage soumise à l'emprise de la religion que la seconde cohorte). À ce propos, voir aussi le commentaire de Danielle Gauvreau et Peter Gossage, loc. cit., 501-510. À tout prendre, l'influence de la religion catholique peut difficilement être niée; il est maintenant bien établi, par exemple, que les populations catholiques d'Europe ont connu leur révolution contraceptive après les populations protestantes; voir Ansley J. Coale et Susan Cotts Watkins, The Decline of Fertility in Europe (Princeton, NJ, Princeton University Press, 1986). Pour le Québec, les données comparées de R. Marvin McInnis, «The Geographic Pattern of Fertility Decline in Canada : 1891-1931», Communication au congrès annuel de la Canadian Association of Geographers, Hamilton, Ontario, 1987; et de R. Marvin McInnis, «Fertility Patterns in Late Nineteenth Century Ontario and Quebec ", Communication au congrès annuel de la Social Science History Association, Chicago, 1988, sont éloquentes également sous ce rapport. 
palement responsable de la moralité dans le couple. C'était sa faute, plus qu'au conjoint, s'il advenait que la famille ne soit pas assez nombreuse. De même, comme nous l'avons signalé, un mari infidèle était le fait d'une épouse qui ne remplissait pas ses devoirs (elle ne lui donnait pas «son besoin»). La plupart des femmes avaient intériorisé cette règle : elles se sentaient coupables si leur mari allait «chercher l'affection ailleurs» (car «il avait bien droit à ses satisfactions», "ç’aurait été trop dur pour lui», «il fallait sauvegarder le foyer», etc.). La communauté leur faisait porter la honte de l'adultère du conjoint. Autre exemple, dans la même veine : si le couple avait fait l'amour «à côté», c'était souvent la femme plutôt que l'homme qui s'en accusait au confessionnal. Nos données contiennent aussi des références à des époux qui auraient voulu pratiquer une forme ou l'autre de contraception, mais qui se heurtaient à un refus de la part de la femme, apeurée par le prêtre et la menace de l'enfer. En somme, et en ce sens, l'homme contrôlait la sexualité mais la procréation relevait davantage de la femme. C'est dire que, par des voies diverses, l'Église saguenayenne a bel et bien contribué, d'une manière significative sans doute, à prolonger le régime de fécondité naturelle en rendant plus difficile le recours à tout moyen contraceptif.

\section{BILAN ET ESSAI DE COMPARAISON}

Résumons d'abord les principaux résultats de notre enquête. La sexualité et la procréation étaient souvent pour la femme synonymes de rudesse et même de violence sur les plans physique et psychologique. En témoignent l'ignorance dans laquelle étaient tenues les adolescentes menstruées, les nouvelles mariées, les mères à leur première grossesse; ou les travaux physiques que s'imposaient les femmes enceintes, les naissances à répétition, les conditions de l'accouchement et, en cas de complications, la priorité inconditionnelle qui était accordée à la vie de l'enfant. On pourrait parler ici, sans exagération, d'une forme d'aliénation inhérente à la condition féminine. Dans ces circonstances, on pourra s'étonner un peu que des informatrices aient pu déclarer après coup (le recul du temps aidant sans doute) s'être néanmoins épanouies dans leurs rôles de conjointe et de mère. Nous avons vu en outre que les taux élevés de fécondité, tels que mesurés avec les outils les plus sensibles de l'analyse démographique, n'étaient pas incompatibles avec le fait généralisé d'une volonté de réduction de la procréation et même avec l'existence de pratiques contraceptives - si peu raffinées fussent-elles. Cette réalité montre, s’il en est besoin, la place indispensable que doit 
occuper l'histoire culturelle (et qualitative) à côté de l'histoire démographique. Le recours à des techniques inefficaces, dont les effets ne sont guère mesurables, ne doit pas masquer l'existence d'une volonté et de pratiques contraceptives. Dans cette mesure, il paraît nécessaire de distinguer soigneusement entre, d'un côté, la réduction de la fécondité comme réalité démographique et, de l'autre, la contraception comme réalité socioculturelle ${ }^{63}$.

Quant à la relation homme-femme dans la vie conjugale, nous avons vu qu'elle consacrait la domination de l'homme en matière de sexualité. Celle-ci faisait même l'objet d'un enseignement et d'une réglementation spécifiques pour la femme. Sur cette base, une historiographie d'inspiration féministe ${ }^{64}$ a mis de l'avant une nouvelle voie d'analyse de la fécondité qui attache beaucoup d'importance au rapport entre les deux conjoints. Par exemple, un rapport patriarcal dans la vie conjugale était de nature à faire obstacle à l'utilisation des moyens contraceptifs faisant appel à la coopération entre les deux partenaires (le retrait, la méthode rythmique, l'abstinence...). Ce facteur a son importance, assurément, mais il a tout de même une portée limitée. En effet, il subsistait toujours un éventail de moyens accessibles à la femme, même sans la coopération de son partenaire. Au Saguenay, c'était le cas avec l'allaitement prolongé, les moyens pour expulser le sperme du vagin, pour le détruire ou pour obstruer l'accès à l'utérus, toutes les techniques de rejet de l'embryon, l'avortement prématuré... En conséquence, une relation conjugale de type patriarcal est un facteur très significatif, certes, mais il porte un peu à confusion, dans la mesure où il peut rendre compte de la vie sexuelle bien plus que de la procréation comme telle ${ }^{65}$. Or, cette distinction est

63. Voir à ce sujet Timothy W. Guinnane, Barbara S. Okun, James Trussell, «What Do We Know About the Timing of Fertility Transitions in Europe?», Demography, 31,1 (février 1994) : 120. Nous remercions Danielle Gauvreau qui a attiré notre attention sur ce texte.

64. Daniel Scott Smith, "Family Limitation, Sexual Control and Domestic Feminism in Victorian America", Feminist Studies, 1 (1973) : 40-57; Carl N. Degler, At Odds: Women and the Family in America from the Revolution to the Present (New York, Oxford University Press, 1980); Nancy R. Folbre, "Of Patriarchy Born: The Political Economy of Fertility Decisions ", Feminist Studies, 9,2 (1983) : 261-284; Susan A. McDaniel, «Une approche sociologique féministe pour l'étude de la fécondité », dans Hubert Gérard et Victor Piché, dir., La sociologie des populations (Montréal, Presses de l’Université de Montréal, 1995), 155-170; Alison Mackinnon, «Were Women Present at the Demographic Transition? Questions from a Feminist Historian to Historical Demographers ", Gender \& History, 7,2 (août 1995) : 222-240; etc.

65. En ce qui concerne le pouvoir que l'homme détenait dans l'ensemble du rapport conjugal, nous avons montré ailleurs qu'il reposait bien plus sur les arrangements sociétaux du droit et de 
essentielle. Dans les campagnes saguenayennes, si c'est l'homme surtout qui contrôlait la sexualité, comme nous l'avons souligné, la procréation semble avoir relevé davantage de la femme. Des moyens contraceptifs lui étaient accessibles, mais elle n'y avait pas recours à cause de ses croyances religieuses, à cause de la crainte du prêtre et de la communauté. Et si elle en faisait usage néanmoins, c'est surtout elle qui portait le poids de la faute. Même si plusieurs de ces moyens étaient inefficaces, il n’empêche que ces femmes pouvaient avoir le sentiment d'un pouvoir réel et surtout d'une lourde responsabilité en matière contraceptive, que venait accentuer le discours clérical. En définitive, croyons-nous, le refus de la contraception découlait moins de la domination masculine dans le couple (laquelle a par ailleurs joué un rôle incontestable) que de contraintes macrosociales représentées par le pouvoir de l'Église et de l'État. Ou, si l'on veut : le patriarcat mais à l'échelle sociétale ${ }^{66}$.

À la lumière de ces remarques, on perçoit l'importance du déclin du sentiment religieux eu égard à l'évolution des taux de fécondité. La désaffection à l'endroit du catholicisme au Saguenay, surtout à partir des années 1940-1950, a supprimé un frein important à la généralisation des pratiques contraceptives et au choix des moyens utilisés. Enfin, nos données établissent aussi - dans ce cadre régional tout au moins - le caractère inapproprié de la distinction qui est souvent faite au sein des moyens contraceptifs entre ceux que l’Église permettait parce qu'ils étaient jugés naturels (allaitement, abstinence...) et ceux qu'elle condamnait sous prétexte qu'ils étaient artificiels et offensaient la nature (gelées et mousses, condoms, avortement...). En réalité, avant 1930, l’Église interdisait tous ces moyens indistinctement, ne faisant exception que dans des circonstances très particulières.

Sur le plan social, les femmes trouvaient souvent des alliés parmi les élites, en l'occurrence les médecins, et parfois au sein du clergé où on pouvait toujours trouver des prêtres plus indulgents que d'autres. Dans le cas contraire, la femme obtempérait aux règles mais n'en nourrissait pas

la religion que sur un ascendant personnel acquis dans l'espace microsocial de la vie matrimoniale (Gérard Bouchard, "Through the Meshes...»).

66. Dans une perspective marxiste, on a pu faire valoir en outre que la classe capitaliste avait également intérêt à ce que se reproduise une force de travail nombreuse et peu coûteuse (par exemple : Nancy R. Folbre, loc. cit.). Ce genre d'interprétation convient sans doute mieux au milieu industriel qu'à une société de petits propriétaires paysans. Il est vrai que dans un contexte de peuplement, la famille nombreuse était utile mais, dans le cas du Saguenay, l'analyse statistique a bien montré que la fécondité n'était pas d'abord commandée par l'écologie de la terre (Gérard Bouchard, Quelques arpents..., op. cit., chap. XIX). 
moins, dans la majorité des cas, des dispositions réfractaires qu'on voit s'exprimer dans la conviction avec laquelle elle essayait de protéger ce territoire réservé - ce no man's land - qu'était la période des relevailles, dans les vaines tentatives pour repousser la prochaine grossesse, dans la volonté d'échapper un tant soit peu aux interventions inquisitrices du prêtre. De toute évidence, «chant de femme» et «sermon de prêtre» ne s'accordaient pas aussi harmonieusement que l'a dit Louis Hémon dans Maria Chapdelaine.

Malgré toutes les contraintes et les tabous qui l'entouraient, on constate que, dès avant 1930, la sexualité avait néanmoins commencé à émerger comme catégorie et comme pratique socioculturelle. Un peu paradoxalement, c'est dans les mois qui suivaient le début de la grossesse que les couples (la femme surtout) pouvaient jouir un peu plus librement de la sexualité. Pour reprendre l'expression d'une informatrice, c'est une période durant laquelle "on se lâchait lousse» (corpus G. Bouchard). Il en était ainsi, et davantage encore, des années qui suivaient la ménopause et que plusieurs femmes ont dit avoir vécues comme une délivrance. De l'ensemble des données orales se dégage l'impression que les couples accédaient alors à un certain affranchissement et à une plus grande sérénité, en particulier parce que la femme, qui ne représentait plus le même enjeu moral, ne faisait plus l'objet d'une étroite surveillance de la part de l'Église. Cela dit, il ne faut voir en tout cela que l'amorce d'une importante évolution qui survint après notre période et qui ne fut pas complétée avant les années 1960, soit la dissociation à peu près totale de la sexualité et de la procréation.

Pour le reste, la plupart des changements significatifs ou annonciateurs de la modernité sont survenus après les années 1930 dans cette région, qu'il s'agisse de la réduction de la fécondité, de l'introduction de nouveaux moyens contraceptifs (thermomètre, calendrier...), de la tolérance accrue de la part de l'Église, d'un certain relâchement des mours (vestimentaires notamment : robes et manches plus courtes), d'un déclin de l'allaitement, d'une désaffection à l'égard de la religion et de l'Église. Certains prêtres commencèrent à parler de la méthode rythmique au confessionnal, à donner des «informations » aux mères en difficulté. Dans les années 1940, le curé Bluteau de Saint-Félicien (Lac-Saint-Jean) expliquait aux femmes dont la santé était compromise comment éviter les grossesses ${ }^{67}$. Des "petits livres" suspects commencèrent à circuler en 
cachette. Un couple mentionne s'être procuré de «la crème des États» (corpus M. St-Hilaire). Un autre utilisait «une petite fiole» venant également des États-Unis (corpus G. Bouchard). En gros, ces changements paraissent en consonance avec la chronologie proposée par Gaston Desjardins ${ }^{68}$, qu'il s'agisse de l'évolution des pratiques sexuelles ou du discours de l'Église catholique sur la sexualité.

Ce dernier énoncé appellerait toutefois bien des nuances que seule une comparaison avec d'autres régions du Québec rendrait possibles. Or, un tel exercice se heurte présentement à la rareté des travaux historiques sur le sujet. En outre, les quelques études disponibles au Québec (à l'extérieur du Saguenay) portent sur le milieu urbain ${ }^{69}$ ou sur l'ensemble de la société québécoise ${ }^{70}$. Il faut donc se contenter de quelques aperçus un peu biaisés du fait qu'ils confrontent principalement les couples paysans du Saguenay avec des couples citadins montréalais et autres. Mais, même dans ces conditions, la comparaison demeure instructive, d'abord par l'importance des similitudes relevées. La caractéristique la plus répandue concerne la grande ignorance des jeunes gens, et en particulier des jeunes filles, en matière de sexualité. Ce trait a été clairement attesté par D. Girard ${ }^{71}$ au sein de trois échantillons de couples montréalais (appartenant à la bourgeoisie, à la classe moyenne et à la classe ouvrière) mariés entre 1920 et 1940. Une observation identique a été faite par M.-J. Boisvert ${ }^{72}$, pour la même période, dans un échantillon de couples ouvriers de Trois-Rivières ${ }^{73}$. Enfin, le phénomène a été relevé également par D. Baillargeon ${ }^{74}$ et par D. Lemieux

68. Gaston Desjardins, «La pédagogie du sexe : un aspect du discours catholique sur la sexualité au Québec (1930-1960)", Revue d'histoire de l'Amérique française, 43,3 (hiver 1990) : 381-401; L'amour en patience...; «Une mémoire hantée. L'histoire de la sexualité au Québec», Cap-auxDiamants, 49 (printemps 1997) : 10-14.

69. Par exemple : Denyse Baillargeon, op. cit.; Andrée Lévesque, op. cit.

70. Serge Gagnon, op. cit.; Denise Lemieux et Lucie Mercier, op. cit.

71. Denise Girard, Différenciation sociale et rituels du mariage : les Montréalais francophones, 1925 1940, thèse de doctorat (ethnologie des francophones en Amérique du Nord), Université Laval, 1997, 305 ; Mariage et classes sociales : les Montréalais francophones entre les deux Guerres (Sainte-Foy, Les Presses de l'Université Laval/Les Éditions de l'IQRC, 2000).

72. Marie-Josée Boisvert, Les rituels du mariage des ouvriers de Trois-Rivières, 1925-1940, mémoire de maîtrise (études québécoises), Université du Québec à Trois-Rivières, 1996.

73. Il s'agit, dans le premier cas, d'une thèse de doctorat et, dans le second cas, d'un mémoire de maitrise réalisés dans le cadre du projet de recherche de l'IREP sur les dynamiques culturelles interrégionales au Québec. Dans le même sens, voir la thèse de doctorat de Martine Tremblay réalisée au sein du même projet : Les rituels du mariage dans la vallée du Haut-Richelieu au XXe siècle, indicateurs de la différenciation sociale, thèse de doctorat (études québécoises), Université du Québec à Trois-Rivières, 1998, $326 \mathrm{p}$.

74. Denyse Baillargeon, op. cit., chap. 7. 
et L. Mercier ${ }^{75}$. On voit là aussi que c'est le prêtre qui, à l'occasion, instruisait les futures mariées sur les choses du sexe. La méconnaissance des moyens contraceptifs un peu sophistiqués semble avoir été généralisée jusque dans les années 1930. Tout ce qui concernait la procréation et l'enfantement était entouré de tabous, les grossesses étaient cachées le plus longtemps possible, et le reste. La morale sexuelle était aussi très sévère, notamment au chapitre des fréquentations ${ }^{76}$, et la religion était hautement respectée. Par exemple, si l'on en juge par les entrevues effectuées par D. Baillargeon et réanalysées par D. Gauvreau et P. Gossage ${ }^{77}$, il semble que les plus vieux couples consultés se soient montrés très réfractaires à utiliser des moyens contraceptifs interdits par l'Église, même si ces moyens comptaient parmi les plus efficaces à l'époque.

En ce qui concerne les différences, l'abandon de l'allaitement paraît avoir été plus précoce et plus rapide à Montréal ${ }^{78}$. On sait par ailleurs, grâce à A. et A. T. McLaren ${ }^{79}$ et d'autres, qu'on pouvait assez aisément s'y procurer des condoms et autres contraceptifs de nature mécanique dans les années 1930 (plus tôt encore dans le cas des condoms). L'avortement, l'infanticide et l'abandon d'enfants n'y étaient pas inconnus non plus ${ }^{80}$. Quant à la baisse de la fécondité chez les couples francophones, elle est perceptible dès les dernières décennies du $\mathrm{XIX}^{\mathrm{e}}$ siècle dans les comtés les plus urbanisés de l'ouest du Québec ${ }^{81}$. L'enquête sur la fécondité québécoise réalisée par J. Henripin et É. Lapierre-Adamcyk ${ }^{82}$ l'atteste également $^{83}$, de même que les entrevues réalisées par D. Baillargeon ${ }^{84}$. Nous avons vu que la baisse de la fécondité est survenue plus tardivement dans la région du Saguenay.

75. Denise Lemieux et Lucie Mercier, op. cit., 154, passim.

76. Ici, encore une fois, les nombreuses entrevues (quelque 300) réalisées par l'IREP sur le rituel du mariage dans une dizaine de régions du Québec (dans le cadre du projet sur les dynamiques culturelles interrégionales) fournissent des témoignages tout à fait concordants, en milieu urbain comme en milieu rural.

77. Danielle Gauvreau et Peter Gossage, loc. cit.

78. Denyse Baillargeon, «L’encadrement de la maternité au Québec entre les deux guerres : les gardes de La Métropolitaine, les Gouttes de lait et l'Assistance maternelle ", Bulletin RCHTQ, 47-48 (été-automne 1990) : 19-45.

79. Angus McLaren et Arlene Tiger McLaren, op. cit., 21.

80. Andrée Lévesque, op. cit., chap. V.

81. R. Marvin McInnis, "The Geographic Pattern... »; "Fertility Patterns in Late...».

82. Jacques Henripin, Évelyne Lapierre-Adamcyk, op. cit.

83. Voir aussi la réutilisation qui a été faite de ces données par Danielle Gauvreau et Peter Gossage, loc. cit.

84. Cependant, dans les entrevues qu'elle a effectuées au cours des dernières années auprès de paysans âgés de quatre paroisses de la vallée du Saint-Laurent, Diane Gervais a trouvé peu de 
Enfin, l'ouvrage de H. Miner ${ }^{85}$ sur la paroisse de Saint-Denis (Kamouraska) offre quelques éléments de comparaison avec le milieu rural, mais pour les années 1930 seulement. On y apprend que l'allaitement prolongé était alors en déclin, que des techniques d'avortement étaient connues et que des jeunes qui avaient séjourné à Québec ou à Montréal étaient informés de certains moyens contraceptifs. Mais Miner ne s'est pas davantage intéressé à la procréation et à la sexualité des paysans — ou, tout simplement, il n'a pas pu appréhender cet aspect très secret de la vie conjugale (il maîtrisait peu le français et ne pouvait communiquer directement avec les habitants) ${ }^{86}$.

Il faut en convenir : le bilan de cet exercice comparatif est plutôt maigre et il y a peu de chance désormais que, pour une période aussi ancienne, d'autres corpus de données orales puissent être constitués, la plupart des témoins étant maintenant décédés. Il en ressort malgré tout quelques données importantes, tout particulièrement l'ignorance générale en matière de sexualité, la grande sévérité morale et l'influence importante de la religion aussi bien en milieu métropolitain que dans une paysannerie périphérique. Ces traits permettent d'insérer le Saguenay et le Québec dans un panorama bien plus large qui embrasse en réalité tout l'Occident. On trouve en effet de nombreuses descriptions analogues pour le Canada anglais, les États-Unis, la Grande-Bretagne, l'Europe continentale $^{87}$. Il faut peut-être s'en étonner un peu : comment expliquer que, dans des environnements religieux et culturels aussi différenciés, une morale sexuelle quasi uniforme ait pu se déployer avec tant de vigueur ${ }^{88}$ ?

trace de contraception; même l'abstinence était condamnée à l'époque où ces couples ont eu leurs enfants (communication personnelle). Ces entrevues ont été réalisées dans le cadre du projet Saint-Laurent au sein de l'IREP (Gérard Bouchard, Diane Gervais, Le projet Saint-Laurent. Problématique et grille d'entrevue. Nouvelle version), Document de l'IREP II-C-231 (1994).

85. Horace Miner, St. Denis : A French-Canadian Parish (Chicago, The University of Chicago Press, 1963), 299. Traduction française : Saint-Denis : un village québécois (LaSalle, Québec, Hurtubise HMH, 1985).

86. Informations recueillies par l'auteur en 1985 auprès de vieux villageois de Saint-Denis (Kamouraska).

87. Aux États-Unis, par exemple, on peut en juger par les résultats de l'enquête Mosher réalisée entre 1890 et 1920. Bien qu'elles aient appartenu à un milieu urbain scolarisé et favorisé économiquement, les 46 femmes interviewées faisaient preuve d'une grande ignorance en matière sexuelle au moment de leur mariage.

88. Nous posons la question tout en sachant à quel point il serait difficile d'y apporter une réponse satisfaisante. Il faudrait pouvoir tenir compte de la grande variabilité, des contrastes qui caractérisaient les grandes régions de l’Europe. La périodisation très inégale des changements socio- 


\section{CONCLUSION}

L'objectif principal de cet essai était d'aborder la sexualité conjugale comme fait et comme rapport social, en tant qu'insérée dans un système d'idéologies, de tensions et de pouvoirs. Et c'est bien ainsi qu'elle s'est révélée. Objet d'enjeux collectifs (politiques, culturels, économiques), la fécondité était sous haute surveillance; l'information était socialement contrôlée; les pratiques sexuelles tombaient sous la double ordonnance du rapport conjugal (dominé par le mari) et du rapport sociétal (dominé par l'Église et l'État); des pressions sociales faisaient obstacle à l'utilisation des moyens contraceptifs et enlevaient au couple - à la femme plus encore la maîtrise de ses actes, ou du moins la restreignaient substantiellement; la moralité et la responsabilité en matière sexuelle n'étaient pas les mêmes pour l'homme et pour la femme, et le reste. La sexualité paysanne : non seulement un rapport social, mais un rapport profondément inégal.

Enfin, il convient d'insister sur la situation complexe et difficile qui était faite à la femme dans un tel contexte social et culturel. D'un côté, comme nous l'avons montré, elle se trouvait en situation au moins virtuelle de pouvoir sous divers rapports, par exemple : transgression de l'interdiction en matière de contraception, subterfuges pour déjouer certains privilèges de l'époux, possibilité de décision en matière de procréation. Mais elle devait faire face aussi à toutes les pressions contraires de la communauté. Elle se trouvait ainsi coincée, occupant le point de jonction entre les impératifs de l'homme (à l'échelle microsociale) et de l'Église (à l'échelle macrosociale). Cette situation était une source de tensions, de déchirement et d'angoisse qui faisait de la femme, non pas un sujet passif de sa condition, mais un acteur fortement brimé, comme l'atteste le maintien du haut niveau de fécondité jusqu'aux premières décennies du $\mathrm{xx}^{\mathrm{e}}$ siècle.

Sous divers aspects, les conclusions de cette enquête viennent confirmer quelques vieux stéréotypes concernant la femme, la sexualité et la religion dans cette société rurale canadienne-française. Dans cette mesure, elles s'accordent mal avec le courant moderniste qui a marqué l'historiographie québécoise depuis quelques décennies - et dans lequel nous avons nous-même inscrit une partie de nos travaux antérieurs. Sur d'autres points, nos analyses ont conduit à de nouveaux aperçus qui remettent en question, cette fois, des représentations courantes en histoire

culturels (laïcisation, scolarisation, déclin de la fécondité...) devrait aussi être prise en compte. Et il resterait encore à surmonter tout le problème des écarts entre les normes et les comportements,etc. Néanmoins, les éléments de similitude et même de synchronie forcent l'attention. 
sociale ou en démographie historique. Tous ces résultats s'accordent avec l'esprit dans lequel nous avons voulu mener cette enquête, en mettant provisoirement à distance les grandes thèses qui balisent actuellement les études historiques sur la sexualité et la condition féminine. Nous nous sommes laissé guider en priorité par nos corpus de données orales, dont nous avons déjà signalé la diversité, l'ampleur et les enseignements convergents. Nous nous en sommes remis aussi au fichier de population BALSAC, dont la fiabilité a été maintes fois démontrée dans le passé, pour tout ce qui concerne notamment l'évolution de la fécondité. Or, la stabilité qui la caractérise jusqu’en 1930 est une donnée lourde; elle suggère que les paramètres sociaux et culturels qui commandent en profondeur les comportements procréatifs n'ont pas beaucoup bougé eux non plus entre 1860 et $1930^{89}$. Enfin, il nous a paru essentiel d'appuyer nos analyses de la sexualité et de la condition féminine sur une connaissance approfondie des structures familiales et de la société rurale saguenayenne dans son ensemble. De ce point de vue, l'ensemble des travaux réalisés sur l'histoire de cette région depuis trente ans (en particulier notre ouvrage Quelques arpents d'Amérique) offraient un recours adéquat. La principale question qui se pose, dans ces conditions, est celle de la généralisation : dans quelle mesure nos résultats sont-ils transposables à d'autres régions, à d'autres contextes? Dans l'état présent de la recherche, il serait téméraire de répondre par l'affirmative - bien que les importantes similitudes et coïncidences révélées par les comparaisons soient troublantes. De même, il serait illégitime de conclure à un exceptionnalisme saguenayen tant que des enquêtes appuyées sur des bases empiriques équivalentes n'auront pas été effectuées ailleurs.

89. Certes, cette enquête montre que des changements sont survenus dans la population paysanne bien avant 1930; mais la stabilité du taux de fécondité autorise à conclure qu'ils n'ont pas altéré ce que nous appelons les paramètres de la procréation. 